\title{
KRONECKER POSITIVITY AND 2-MODULAR REPRESENTATION THEORY
}

\author{
C. BESSENRODT, C. BOWMAN, AND L. SUTTON
}

\begin{abstract}
This paper consists of two prongs. Firstly, we prove that any Specht module labelled by a 2-separated partition is semisimple and we completely determine its decomposition as a direct sum of graded simple modules. Secondly, we apply these results and other modular representation theoretic techniques on the study of Kronecker coefficients and hence verify Saxl's conjecture for several large new families of partitions. In particular, we verify Saxl's conjecture for all irreducible characters of $\mathfrak{S}_{n}$ which are of 2-height zero.
\end{abstract}

\section{INTRODUCTION}

This paper brings together, for the first time, the two oldest open problems in the representation theory of the symmetric groups and their quiver Hecke algebras. The first problem is to understand the structure of Specht modules and the second is to describe the decomposition of a tensor product of two Specht modules - the Kronecker problem.

Kronecker positivity. The Kronecker problem is not only one of the central open problems in the classical representation theory of the symmetric groups, but it is also one of the definitive open problems in algebraic combinatorics as identified by Richard Stanley in Sta00. The problem of deciding the positivity of Kronecker coefficients arose in recent times also in quantum information theory Kly04,CM06, CHM07,CDW12 and Kronecker coefficients have subsequently been used to study entanglement entropy [CSW18.

A new benchmark for the Kronecker positivity problem is a conjecture of Heide, Saxl, Tiep and Zalesskii HSTZ13. that was inspired by their investigation of the square of the Steinberg character for simple groups of Lie type. It says that for any $n \neq 2,4,9$ there is always a complex irreducible character of $\mathfrak{S}_{n}$ whose square contains all irreducible characters of $\mathfrak{S}_{n}$ as constituents. For $n$ a triangular number, an explicit candidate was suggested by Saxl in 2012: Let $\rho:=\rho(k)=(k, k-1, k-$ $2, k-3, \ldots, 2,1)$ denote the $k$ th staircase partition. Phrased in terms of modules, Saxl's conjecture states that all simple modules appear in the tensor square of the simple $\mathbb{C S}_{n}$-module $\mathbf{D}^{\mathbb{C}}(\rho)$. In other words, we have that

$$
\mathbf{D}^{\mathbb{C}}(\rho) \otimes \mathbf{D}^{\mathbb{C}}(\rho)=\bigoplus_{\lambda} g(\rho, \rho, \lambda) \mathbf{D}^{\mathbb{C}}(\lambda)
$$

Received by the editors August 2, 2019, and, in revised form, September 2, 2020.

2020 Mathematics Subject Classification. Primary 05E10, 20C30.

The second author would like to thank both the Alexander von Humboldt Foundation and the Leibniz Universität Hannover for financial support and an enjoyable summer. The third author was supported by Singapore MOE Tier 2 AcRF MOE2015-T2-2-003. 
with $g(\rho, \rho, \lambda) \neq 0$ for all partitions $\lambda$ of $n$. This conjecture has been studied by algebraists, probabilists, and complexity theorists [Bes18, Ike15, LS17, PPV16] yet remains to be proved in general. Positivity of the Kronecker coefficient $g(\rho, \rho, \lambda)$ has been verified for hooks and two-row partitions when $n$ is sufficiently large in PPV16, and then for arbitrary $n$ and $\lambda$ a hook in Ike15, Bes18, or a double-hook partition (i.e., when the Durfee size is 2) in [Bes18, and for any $\lambda$ comparable to $\rho$ in dominance order in Ike15.

This paper begins with the observation that the $\mathbb{k}_{\mathfrak{S}} \mathfrak{S}_{n}$-module $\mathbf{D}^{\mathbb{k}}(\rho)$ is projective over a field $\mathbb{k}$ of characteristic $p=2$, or equivalently, that the character to the Specht module $\mathbf{D}^{\mathbb{C}}(\rho)=\mathbf{S}^{\mathbb{C}}(\rho)$ is the character $\xi^{\rho}$ associated to a projective indecomposable $\mathbb{k}_{\mathfrak{S}} \mathfrak{S}_{n}$-module (via its integral lift to characteristic 0 ). Therefore, the tensor square of $\mathbf{D}^{\mathbb{k}}(\rho)$ is again a projective module, and the square of $\xi^{\rho}$ is the character to a projective module. This allows us to bring to bear the tools of modular and graded representation theory on the study of the Kronecker coefficients. In particular, we deduce that if $\mathbf{D}^{\mathbb{k}}(\lambda)=\mathbf{S}^{\mathbb{k}}(\lambda)$ is a simple Specht module, then all constituents of the projective cover of $\mathbf{D}^{\mathbb{k}}(\lambda)$ must also appear in Saxl's tensorsquare. For example, using this property for the trivial simple module $\mathbf{D}^{\mathbb{k}}((n))$ of $\mathfrak{S}_{n}$ at characteristic 2 gives all characters of odd degree as constituents in the Saxl square; more generally, we will detect all irreducible characters of 2-height 0 as constituents. Our aim is to understand the columns of the 2-modular graded decomposition matrix which are labelled by simple Specht modules and to utilise these results towards Saxl's conjecture.

Modular representation theory. The classification of simple Specht modules for symmetric groups and their Hecke algebras has been a massive undertaking involving over 30 years of work [Jam78, JM96, JM97, JM99, Fay04, Fay05, JLM06, Lyl07, FL09, Fay10,FL13, with some conjectural cases for $e=2$ and $p \neq 2$ still to be verified. The pursuit of a description of semisimple and decomposable Specht modules is similarly old [Jam78] and yet has proven a much more difficult nut to crack. The decomposable Specht modules labelled by hook partitions were characterised by Murphy and Speyer [Mur80, Spe14; the graded decomposition numbers of these Specht modules were calculated by Chuang, Miyachi, and Tan CMT04; the first examples of decomposable Specht modules labelled by non-hook partitions were given by Dodge and Fayers [DF12]; Donkin and Geranios very recently unified and extended these results to certain "framed staircase" partitions [DG18] which we will discuss (within the wider context of "2-separated" partitions) below. It is worth emphasising that for $e>2$, all Specht modules are indecomposable and therefore questions of decomposability and (non-simple) semisimplicity are inherently 2-modular problems.

For $H_{-1}^{\mathbb{C}}(n)$, we show that any Specht module labelled by a 2 -separated partition is semisimple and we completely determine its decomposition as a sum of graded simple modules. Our proof makes heavy use of recent results in the graded representation theory of Hecke and rational Cherednik algebras. We shall denote the quantisations of the Specht and simple modules by $\mathbf{S}_{q}^{\mathbb{k}}(\lambda)$ and $\mathbf{D}_{q}^{\mathbb{k}}(\lambda)$ respectively over $\mathbb{k}$. We completely determine the rows of the graded decomposition matrix of $H_{-1}^{\mathbb{C}}(n)$ labelled by 2-separated partitions; this serves as a first approximation to our goal and subsumes and generalises the results on decomposability and decomposition numbers of Specht modules for hook partitions (belonging to blocks 
of small 2-core) Spe14,CMT04, and results on decomposition numbers of Specht modules in blocks of enormous 2-cores JM96.

Graded decomposition numbers of semisimple Specht modules. The partitions of interest to us (for both Saxl's conjecture and our decomposability classification) are the 2-separated partitions. Such partitions are obtained by taking a staircase partition, $\tau$, and adding 2 copies of a partition $\lambda$ to the right of $\tau$ and 2 copies of a partition $\mu$ to the bottom of $\tau$ in such a way that $\lambda$ and $\mu$ do not touch (except perhaps diagonally). Such partitions, denoted $\tau_{\mu}^{\lambda}$, can be pictured as in Figure 1
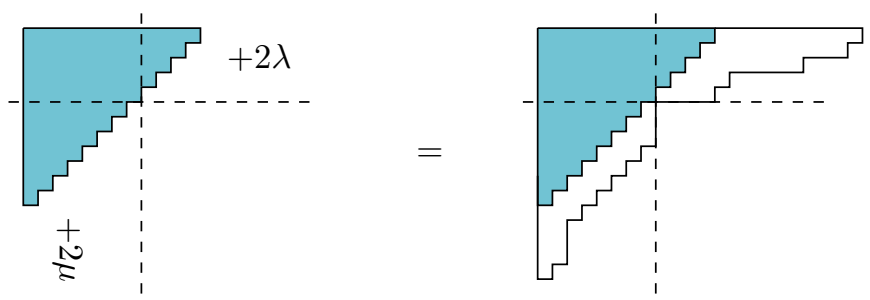

Figure 1. A 2-separated partition $\tau_{\mu}^{\lambda}$ (see Definition 1.6)

Notice that if the weight of a block is small compared to the size of the core, then all partitions in that block are 2-separated. We emphasise that the size of the staircase $\rho(k)$ in the following statement is immaterial (provided that $k+1 \geqslant$ $\ell(\lambda)+\ell\left(\mu^{T}\right)$, where $\ell(\lambda)$ denotes the length of the partition $\lambda$ ), and so we simply write $\tau:=\rho(k)$. For those interested in the extra graded structure, we refer the reader to the full statement in Corollary 4.2 .

Theorem A. Let $\tau_{\mu}^{\lambda}$ denote a 2-separated partition of $n$.

The $H_{-1}^{\mathbb{C}}(n)$-module $\mathbf{S}_{-1}^{\mathbb{C}}\left(\tau_{\mu}^{\lambda}\right)$ is semisimple and decomposes as a direct sum of simples as follows

$$
\mathbf{S}_{-1}^{\mathbb{C}}\left(\tau_{\mu}^{\lambda}\right)=\bigoplus_{\nu} c\left(\nu^{T}, \lambda^{T}, \mu\right) \mathbf{D}_{-1}^{\mathbb{C}}\left(\tau_{\varnothing}^{\nu}\right),
$$

where $c\left(\nu^{T}, \lambda^{T}, \mu\right)$ is the Littlewood-Richardson coefficient labelled by this triple of partitions.

In particular, there exist many blocks of $H_{-1}^{\mathbb{C}}(n)$ (those with large cores) for which all Specht modules in the block are semisimple. In DF12 Dodge and Fayers remark that "every known example of a decomposable Specht module is labelled by a 2-separated partition" and "it is interesting to speculate whether the 2-separated condition is necessary for a Specht module to be decomposable". In fact in Section 6] we show that their speculation is not true by exhibiting two infinite families of decomposable Specht modules obtained by "inflating" the smallest decomposable Specht module (indexed by $\left(3,1^{2}\right)$ ).

Theorem A implies that all known examples of decomposable Specht modules for $\mathfrak{S}_{n}$ are obtained by reduction modulo $p=2$ from decomposable semisimple Specht modules for $H_{-1}^{\mathbb{C}}(n)$. 
Applications to Kronecker coefficients. We now discuss the results and insights which 2-modular representation theory affords us in the study of Kronecker coefficients. We verify the positivity of the Kronecker coefficients in Saxl's conjecture for a large new class of partitions, and propose conjectural strengthened and generalised versions of Saxl's original conjecture. Our first main theorem on Kronecker coefficients is as follows:

Theorem B. Let $\lambda \vdash n=k(k+1) / 2$ such that $\chi^{\lambda}$ is of 2-height 0 . Then $g(\rho, \rho, \lambda)>$ 0 . In particular, all $\chi^{\lambda}$ of odd degree are constituents of the Saxl square.
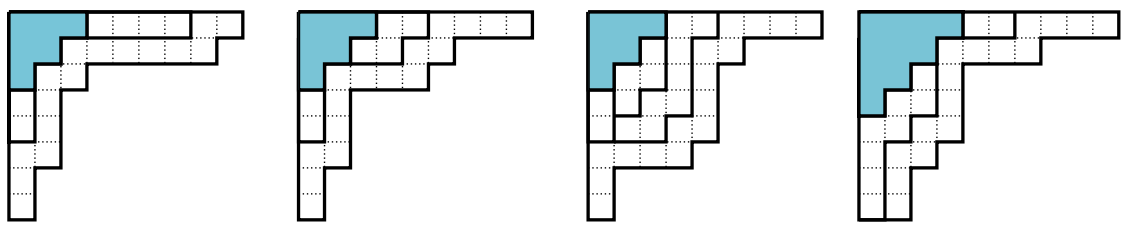

Figure 2. Examples of partitions which label 2-height 0 characters for $\mathfrak{S}_{28}$ and $\mathfrak{S}_{36}$ (and therefore label constituents of Saxl's tensor square by Theorem (B). There are 672 and 1417 such characters for these groups, respectively. A combinatorial construction of all such partitions (for arbitrary $n \in \mathbb{N}$ ) is given in Subsection 1.4 .

We now shift focus to the Kronecker coefficients labelled by 2-separated partitions. In what follows, we shall write $g\left(\rho, \rho, \tau_{\mu}^{\lambda}\right)$ for the Kronecker coefficient labelled by a staircase $\rho$ of size $n=k(k+1) / 2$ for some $k \in \mathbb{N}$ and some 2-separated partition $\tau_{\mu}^{\lambda}$ of $n$; in other words, we do not encumber the notation by explicitly recording the size of the staircases involved.

Theorem C. For $(\alpha, \beta)$ a $k$-Carter-Saxl pair (as in Theorem 5.10) we have that $g(\rho, \rho, \alpha) \geqslant k$. In particular, all framed staircase partitions $\tau_{\left(1^{b}\right)}^{(a)}$ appear in the Saxl square.

We do not recall the definition of a $k$-Carter-Saxl pair here, but rather discuss some examples and consequences of Theorem B. In particular, Theorem B implies that every 2-block contains a wealth of constituents of the Saxl square $\mathbf{S}^{\mathbb{C}}(\rho) \otimes$ $\mathbf{S}^{\mathbb{C}}(\rho)$ which can be deduced using our techniques. Carter-Saxl pairs cut across hook partitions, partitions of arbitrarily large Durfee size, symmetric and nonsymmetric partitions, partitions from arbitrary blocks, and across the full range of the dominance order. (In fact, the only common trait of these partitions is that they label semisimple Specht modules for $H_{-1}^{\mathbb{C}}(n)$.) We shall illustrate below that the property of being a Carter-Saxl pair is actually very easy to work with diagrammatically. For example, the above theorem includes the infinite family of "framed staircases" as some of the simplest examples: these are partitions which interpolate hooks and staircases. More explicitly, these are the partitions of the form $\alpha=\tau_{\left(1^{b}\right)}^{(a)}$. These can be pictured as in Figure 3 below.

We wish to provide bounds on the Kronecker coefficients: the maximal possible values obtained by Kronecker products are studied in PPV16, and the Kronecker products whose coefficients are all as small as possible (namely all 0 or 1) are 

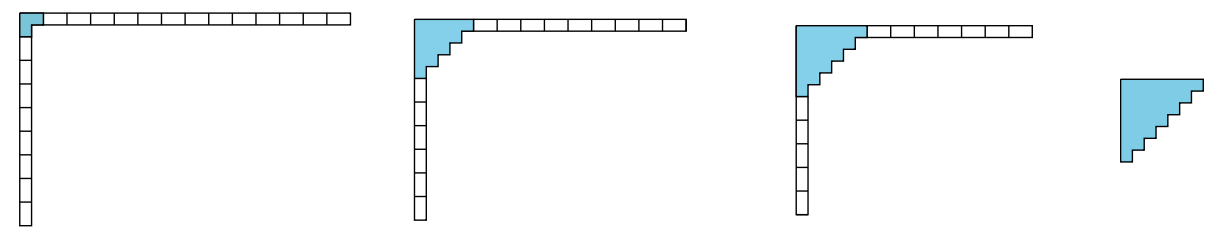

FIGURE 3. Some examples of framed staircases: $\alpha=\tau_{\left(1^{8}\right)}^{(13)}, \tau_{\left(1^{6}\right)}^{(9)}$, $\tau_{\left(1^{5}\right)}^{(7)}$, and $\tau=\rho(9)$ are all partitions of $n=45$. Up to conjugation, there are 35 framed staircase partitions of $n=45$. The classification of decomposable Specht modules labelled by framed staircases is the main result of DG18. In Theorem 5.5 we prove Saxl's conjecture for all framed staircase partitions. The key ingredient in our proof is that $\left(\tau_{\left(1^{b}\right)}^{(a)}, \tau_{\varnothing}^{(a+b)}\right)$ is a 1-Carter-Saxl pair for $a, b \in \mathbb{N}$.

classified in [BB17]. For constituents to partitions of depth at most 4, explicit formulae for their multiplicity in squares were provided by Saxl in 1987, and later work by Zisser and Vallejo, respectively. For the Kronecker coefficients studied here, the easiest (and well known) non-trivial case is $g(\rho(k), \rho(k),(n-1,1))=k-1$, so the Kronecker coefficients are even unbounded; this also holds for the other families corresponding to partitions of small depth. Lower bounds coming from character values on a specific class were obtained by Pak and Panova in [PP17, where also the asymptotic behaviour of the multiplicity of special constituents is studied. Theorem B allows us to provide explicit lower bounds on the Kronecker coefficients $g(\rho(k), \rho(k), \lambda)$ for new infinite families of Saxl constituents, where again the multiplicities are unbounded.

We now provide some examples of more complicated Carter-Saxl pairs. For $n=78$, if we first focus on the (unique) block of weight $w=6$ we find 7 constituents in this block labelled by framed staircases as well as the Carter-Saxl pairs given (up to conjugation) in Figure 4 below.
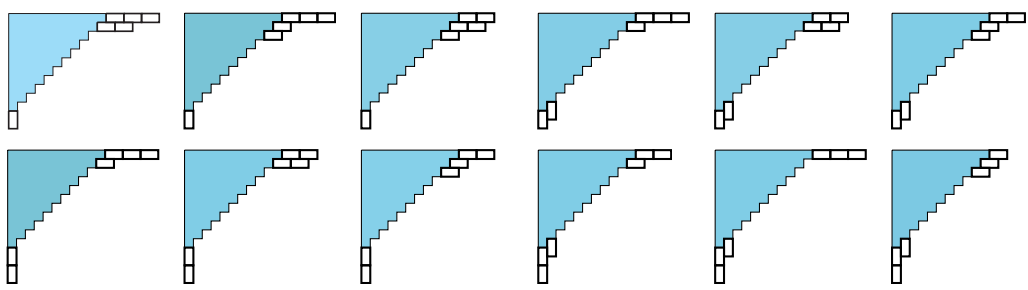

Figure 4. More examples of coefficients $g\left(\rho, \rho, \tau_{\mu}^{\lambda}\right)>0$. These belong to the block of weight 6 for the symmetric group of rank 78. We have that each of $\tau_{\mu}^{\lambda}$ belongs to a Carter-Saxl pair of the form $\left(\tau_{\mu}^{\lambda}, \tau_{\varnothing}^{(3,2,1)}\right)$.

Finally, we propose two extensions of Saxl's conjecture based on its modular representation theoretic interpretation. The first conjecture reduces the problem to the case of 2-regular partitions, but at the expense of working in the more difficult 
modular setting. We remark that towards Saxl's conjecture over $\mathbb{C}$, it has already been verified that for any 2-regular partition $\lambda$ of $n=k(k+1) / 2$ the Kronecker coefficient $g(\rho(k), \rho(k), \lambda)$ is positive [Ike15], and so it is natural to hope that this can be extended to positive characteristic.

Strengthened Saxl Conjecture. Let $\mathbb{k}$ be a field of characteristic 2. We have that

$$
\operatorname{dim}_{\mathbb{k}}\left(\operatorname{Hom}_{\mathfrak{S}_{n}}\left(\mathbf{D}^{\mathbb{k}}(\rho(k)) \otimes \mathbf{D}^{\mathbb{k}}(\rho(k)), \mathbf{D}^{\mathbb{k}}(\lambda)\right)\right)>0
$$

for any 2-regular partition $\lambda$ of $n=k(k+1) / 2$. Equivalently: Saxl's 2-modular tensor square contains all indecomposable projective modules as direct summands with positive multiplicity.

What could be a suitable candidate for arbitrary $n$, not just triangular numbers?

Generalised Saxl Conjecture. For $n \in \mathbb{N}$ there exists a symmetric $p$-core $\lambda$ for some $p \leqslant n$ such that $\mathbf{D}^{\mathbb{C}}(\lambda) \otimes \mathbf{D}^{\mathbb{C}}(\lambda)$ contains all simple $\mathbb{C S}_{n}$-modules with positive multiplicity.

While this sounds reasonable, in fact, for larger $n$ it hardly restricts the search for a good candidate as almost any partition of $n$ is then a $p$-core for some $p \leqslant n$. So as a guide towards finding a simple module $\mathbf{D}^{\mathbb{C}}(\lambda)$ whose tensor square contains all simples, one would try to find a suitable symmetric $p$-core for a small prime $p$.

\section{The Hecke Algebra}

Let $\mathbb{k}$ be a commutative integral domain. We let $\mathfrak{S}_{n}$ denote the symmetric group on $n$ letters, with presentation

$$
\left.\mathfrak{S}_{n}=\left\langle s_{1}, \ldots, s_{n-1}\right| s_{i} s_{i+1} s_{i}=s_{i+1} s_{i} s_{i+1}, s_{i}^{2}=1, s_{i} s_{j}=s_{j} s_{i} \text { for }|i-j|>1\right\rangle .
$$

We are interested in the representation theory (over $\mathbb{k}$ ) of symmetric groups and their deformations. Given $q \in \mathbb{k}$, we define the Hecke algebra $H_{q}^{\mathbb{k}}(n)$ to be the unital associative $\mathbb{k}$-algebra with generators $T_{1}, T_{2}, \ldots, T_{n-1}$ and relations

$$
\left(T_{i}-q\right)\left(T_{i}+1\right)=0 \quad T_{i} T_{j}=T_{j} T_{i}, \quad T_{i} T_{i+1} T_{i}=T_{i+1} T_{i} T_{i+1}
$$

for $|i-j|>1$. We let $e \in \mathbb{N}$ be the smallest integer such that $1+q+q^{2}+\cdots+q^{e-1}=0$ or set $e=\infty$ if no such integer exists. If $\mathbb{k}$ is a field of characteristic $p$ and $p=e$, then $H_{q}^{\mathbb{k}}(n)$ is isomorphic to $\mathbb{k} \mathfrak{S}_{n}$.

We define a composition, $\lambda$, of $n$ to be a finite sequence of non-negative integers $\left(\lambda_{1}, \lambda_{2}, \ldots\right)$ whose sum, $|\lambda|=\lambda_{1}+\lambda_{2}+\ldots$, equals $n$. If the sequence $\left(\lambda_{1}, \lambda_{2}, \ldots\right)$ is weakly decreasing, we say that $\lambda$ is a partition; we denote the set of all partitions of $n$ by $\mathscr{P}_{n}$. The number of non-zero parts of a partition, $\lambda$, is called its length, $\ell(\lambda)$; the size of the largest part is called the width, $w(\lambda)=\lambda_{1}$. Given $\lambda \in \mathscr{P}_{n}$, its Young diagram $[\lambda]$ is defined to be the configuration of nodes,

$$
[\lambda]=\left\{(r, c) \mid 1 \leq r \leq \ell(\lambda), 1 \leqslant c \leqslant \lambda_{r}\right\} .
$$

The conjugate partition, $\lambda^{T}$, is the partition obtained by interchanging the rows and columns of $\lambda$; when $\lambda=\lambda^{T}$, the partition $\lambda$ is said to be symmetric. Given a node $(r, c) \in[\lambda]$ we define the content to be $\operatorname{ct}(r, c)=c-r$ and the $(e-)$ residue to be the value of $\operatorname{ct}(r, c)$ modulo $e$. We now recall the dominance ordering on partitions. Let $\lambda, \mu$ be partitions. We write $\lambda \triangleq \mu$ if

$$
\sum_{1 \leqslant i \leqslant k} \lambda_{i} \geqslant \sum_{1 \leqslant i \leqslant k} \mu_{i} \text { for all } k \geqslant 1
$$


If $\lambda \unrhd \mu$ and $\lambda \neq \mu$ we write $\lambda \triangleright \mu$. For $\lambda, \nu$ partitions such that $\lambda \subseteq \nu$, we define the skew diagram, denoted $[\nu \backslash \lambda]:=[\nu] \backslash[\lambda]$, to be the set difference between the Young diagrams of $\lambda$ and $\nu$.

Given $\lambda \in \mathscr{P}_{n}$, we define a tableau of shape $\lambda$ to be a filling of the nodes of the Young diagram of $\lambda$ with the numbers $\{1, \ldots, n\}$. We define a standard tableau to be a tableau in which the entries increase along both the rows and columns of each component. We let $\operatorname{Std}(\lambda)$ denote the set of all standard tableaux of shape $\lambda \in \mathscr{P}_{n}$. We extend this to (standard) skew tableaux of shape $\nu \backslash \lambda$ in the obvious fashion. Given $\mathrm{t} \in \operatorname{Std}(\lambda)$, we set Shape(t) $=\lambda$. Given $1 \leqslant k \leqslant n$, we let $t_{\{1, \ldots, k\}}$ be the subtableau of $\mathrm{t}$ whose entries belong to the set $\{1, \ldots, k\}$. We write $\mathrm{t} \geqslant \mathrm{s}$ if $\mathrm{t} \downarrow_{\{1, \ldots, k\}} \geqslant \mathrm{s} \downarrow_{\{1, \ldots, k\}}$ for all $1 \leqslant k \leqslant n$ and refer to this as the dominance order on $\operatorname{Std}(\lambda)$.

We let $\mathrm{t}^{\lambda}$ and $\mathrm{t}_{\lambda}$ denote the most and least dominant tableaux respectively. We let $w_{\lambda} \in \mathfrak{S}_{n}$ be the permutation such that $w_{\lambda} \mathrm{t}^{\lambda}=\mathrm{t}_{\lambda}$. For example, $w_{(3,2,1)}=$ $(2,4)(3,6)$ and

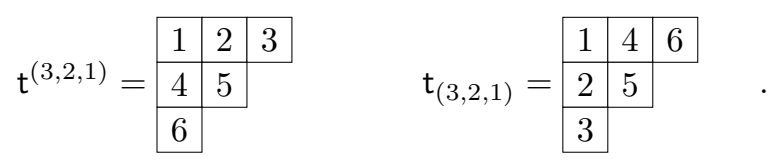

Definition 1.1. Given $\lambda$ a partition of $n$, we set $\mathfrak{S}_{\lambda}=\mathfrak{S}_{\lambda_{1}} \times \mathfrak{S}_{\lambda_{2}} \ldots \leqslant \mathfrak{S}_{n}$ and we set

$$
x_{\lambda}=\sum_{w \in \mathfrak{S}_{\lambda}} T_{w} \quad y_{\lambda}=\sum_{w \in \mathfrak{S}_{\lambda}}(-q)^{\ell(w)} T_{w}
$$

and we define the Specht module, $\mathbf{S}_{q}^{\mathbb{k}}(\lambda)$, to be the left $H_{q}^{\mathbb{k}}(n)$-module

$$
\mathbf{S}_{q}^{\mathbb{k}}(\lambda):=H_{q}^{\mathbb{k}}(n) y_{\lambda} T_{w_{\lambda}} x_{\lambda} .
$$

Remark 1.2. Letting $\mathbb{k}=\mathbb{C}$ and specialising $q=1$ we have that $H_{q}^{\mathbb{k}}(n)$ is isomorphic to $\mathbb{C S}_{n}$. In this case, we drop the subscript on the Specht modules and we have that

$$
\left\{\mathbf{S}^{\mathbb{C}}(\lambda) \mid \lambda \in \mathscr{P}_{n}\right\}
$$

provide a complete set of non-isomorphic simple $\mathbb{C S}_{n}$-modules. We let $\chi^{\lambda}$ denote the character of the complex irreducible module $\mathbf{S}^{\mathbb{C}}(\lambda)$.

1.1. Modular representation theory. Let $\mathbb{k}$ be a field and $q \in \mathbb{k}$. The group algebra of the symmetric group $\mathbb{k} \mathfrak{S}_{n}$ is a semisimple algebra if and only if $\mathbb{k}$ is a field of characteristic $p>n$. By a result of Dipper and James, the Hecke algebra of the symmetric group is a non-semisimple algebra if $q$ is a primitive eth root of unity for some $e \leqslant n$ or $q=1$ and $\mathbb{k}$ is a field of characteristic $p \leqslant n$. We shall now recall the basics of the non-semisimple representation theory of these algebras.

Modular representation theory seeks to deconstruct the non-semisimple representations of an algebra in terms of their simple constituents. To this end, we define the radical of a finite-dimensional $A$-module $M$, denoted $\operatorname{rad}(M)$, to be the smallest submodule of $M$ such that the corresponding quotient is semisimple. We then let $\operatorname{rad}^{2} M=\operatorname{rad}(\operatorname{rad} M)$ and inductively define the radical series, $\operatorname{rad}^{i} M$, of $M$ by $\operatorname{rad}^{i+1} M=\operatorname{rad}\left(\operatorname{rad}^{i} M\right)$. We have a finite chain

$$
M \supset \operatorname{rad}(M) \supset \operatorname{rad}^{2}(M) \supset \cdots \supset \operatorname{rad}^{i}(M) \supset \operatorname{rad}^{i+1}(M) \supset \cdots \supset \operatorname{rad}^{s}(M)=0 .
$$

In the non-semisimple case, the Specht modules are no longer simple but they continue to play an important role in the representation theory of $H_{q}^{\mathbb{k}}(n)$ as we 
shall now see. We say that a partition $\lambda=\left(\lambda_{1}, \lambda_{2}, \ldots, \lambda_{\ell}\right)$ is $e$-regular if there is no $1 \leqslant i \leqslant \ell$ such that $\lambda_{i}=\lambda_{i+1}=\cdots=\lambda_{i+e-1}>0$. We let $\mathscr{R}_{n}^{e}$ denote the set of all $e$-regular partitions of $n$. Occasionally, we will also use the notation $\lambda \vdash_{e} n$ in place of $\lambda \in \mathscr{R}_{n}^{e}$. For $\mathbb{k}$ an arbitrary field, we have that

$$
\left\{\mathbf{D}_{q}^{\mathbb{k}}(\mu) \mid \mathbf{D}_{q}^{\mathbb{k}}(\mu)=\mathbf{S}_{q}^{\mathbb{k}}(\mu) / \operatorname{rad}\left(\mathbf{S}_{q}^{\mathbb{k}}(\mu)\right), \mu \in \mathscr{R}_{n}^{e}\right\}
$$

provides a full set of non-isomorphic simple $H_{q}^{\mathbb{k}}(n)$-modules. Of course, the radical of a Specht module is not easy to compute! The passage between the Specht and simple modules is recorded in the decomposition matrix,

$$
\left(d_{\lambda \mu}^{\mathbb{k}}\right)_{\substack{\lambda \in \mathscr{P}_{n} \\ \mu \in \mathscr{R}_{n}^{e}}} \quad d_{\lambda \mu}^{\mathbb{k}}=\left[\mathbf{S}_{q}^{\mathbb{k}}(\lambda): \mathbf{D}_{q}^{\mathbb{k}}(\mu)\right],
$$

where $\left[\mathbf{S}_{q}^{\mathbb{k}}(\lambda): \mathbf{D}_{q}^{\mathbb{k}}(\mu)\right]$ denotes the multiplicity of $\mathbf{D}_{q}^{\mathbb{k}}(\mu)$ as a composition factor of $\mathbf{S}_{q}^{\mathbb{k}}(\lambda)$. This matrix is uni-triangular with respect to the dominance ordering on $\mathscr{P}_{n}$. We have already seen in equation (1.1) that every column of the decomposition matrix contains an entry equal to 1 ; namely if $\mu \in \mathscr{R}_{n}^{e}$ then $d_{\mu, \mu}=1$. We now recall James' regularisation theorem, which states that every row of the decomposition matrix contains an entry equal to 1 (and identifies this entry).
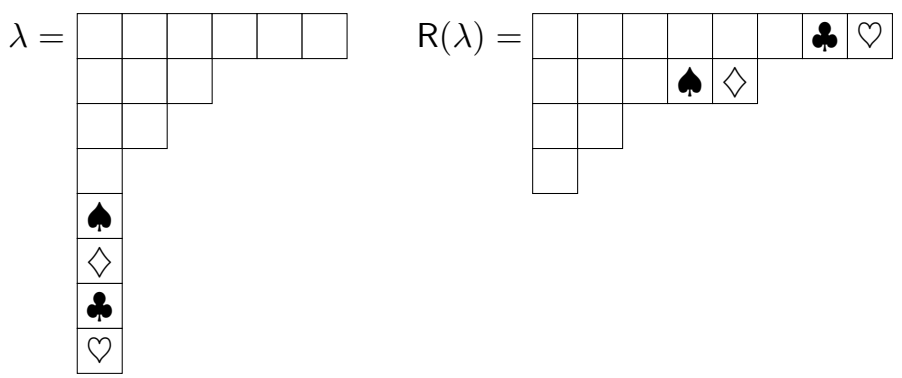

FIgURE 5. The partition $\lambda=\tau_{\left(1^{2}\right)}^{(1)}(4)$, its 2-regularisation $\mathrm{R}(\lambda)$

Example 1.3. We picture a partition $\lambda$ and its 2-regularisation $\mathrm{R}(\lambda)$ in Figure 5 , We have highlighted which nodes are moved and to where they have been moved.

We define the $(e-)$ ladder number of a node $(r, c) \in[\lambda]$ to be $\mathfrak{l}(r, c)=r+c(e-1)$. The $i$ th ladder of $\lambda$ is defined to be the set

$$
\mathscr{L}_{i}=\left\{(r, c) \in \mathbb{N}^{2} \mid \mathfrak{l}(r, c)=i\right\} \cap[\lambda] .
$$

The $e$-regularisation of $\lambda$ is the partition $\mathrm{R}(\lambda)$ obtained by moving all of the nodes of $\lambda$ as high along their ladders as possible. When $q=-1$, each ladder of $\lambda$ is a complete north-east to south-westerly diagonal in $[\lambda]$. In particular, when $e=2$ the partition $\mathrm{R}(\lambda)$ is obtained from $\lambda$ by sliding nodes as high along their south-west to north-easterly diagonals as possible.

Theorem 1.4 (James' regularisation theorem). Let $\lambda$ be a partition of $n$ and $\mathbb{k}$ be an arbitrary field. We have that $\left[\mathbf{S}_{q}^{\mathbb{k}}(\lambda): \mathbf{D}_{q}^{\mathbb{k}}(\mu)\right]$ is equal to 1 if $\mu=\mathrm{R}(\lambda)$ and is zero unless $\mu \geqslant \mathrm{R}(\lambda)$. 
1.2. Brauer-Humphrey's reciprocity. Given $\lambda$ an $e$-regular partition and $\mathbf{D}_{q}^{\mathbb{k}}(\lambda)$ the corresponding simple $H_{q}^{\mathbb{k}}(n)$-module, we let $\mathbf{P}_{q}^{\mathbb{k}}(\lambda)$ denote its projective cover. Brauer-Humphrey's reciprocity states that $\mathbf{P}_{q}^{\mathbb{k}}(\mu)$ has a Specht filtration,

$$
0=S_{1} \subset S_{2} \subset \cdots \subset S_{z}=\mathbf{P}_{q}^{\mathbb{k}}(\mu)
$$

such that for each $1 \leqslant r \leqslant z$, we have $S_{r} / S_{r-1} \cong \mathbf{S}_{q}^{\mathbb{k}}(\lambda)$ for some $\lambda \in \mathscr{P}_{n}$ dependent on $1 \leqslant r \leqslant z$ and such that the multiplicity, $\left[\mathbf{P}_{q}^{\mathbb{k}}(\mu): \mathbf{S}_{q}^{\mathbb{k}}(\lambda)\right]$, in this filtration is given by

$$
\left[\mathbf{P}_{q}^{\mathbb{k}}(\mu): \mathbf{S}_{q}^{\mathbb{k}}(\lambda)\right]=\left[\mathbf{S}_{q}^{\mathbb{k}}(\lambda): \mathbf{D}_{q}^{\mathbb{k}}(\mu)\right] .
$$

In other words, the $\lambda$ th column of the decomposition matrix determines the multiplicities in a Specht filtration of $\mathbf{P}_{q}^{\mathbb{k}}(\lambda)$. This will be a key observation for our applications to Kronecker coefficients in Section 5 .

1.3. 2-blocks. We first recall the block-structure of Hecke algebras in (quantum) characteristic $e=2$ (which will be the main case of interest in this paper). Throughout this section $e=2$ and $\mathbb{k}$ can be taken to be an arbitrary field (although we are mainly interested in the cases when $\mathbb{k}=\mathbb{C}$ or $\mathbb{k}$ is of characteristic $p=2$ ). The algebra $H_{-1}^{\mathbb{k}}(n)$ decomposes as a direct sum of primitive 2-sided ideals, called blocks. All questions concerning modular representation theory break-down block-by-block according to this decomposition: in particular each simple/Specht module belongs to a unique block.

The rim of the Young diagram of $\lambda \vdash n$ is the collection of nodes $R[\lambda]=\{(r, c) \in$ $[\lambda] \mid(r+1, c+1) \notin[\lambda]\}$. Given $(r, c) \in[\lambda]$, we define the associated rim-hook to be the set of nodes $h(r, c)=\{(i, j) \in R[\lambda] \mid r \leqslant i, c \leqslant j\}$. If $|h(r, c)|=e \in \mathbb{N}$, then we refer to $h$ as a removable $e$-hook; if $e=2$ we refer to $h(r, c)$ as a removable domino. Removing $h(r, c)$ from $[\lambda]$ gives the Young diagram $[\lambda] \backslash h(r, c)$ of a partition of $n-e$. It is easy to see that a partition has no removable dominoes if and only if it is of the form $\rho(k)=(k, k-1, k-2, \ldots, 2,1)$ for some $k \geqslant 0$, in which case we say that it is a 2-core. We let core $(\lambda)$ denote the 2 -core partition obtained by successively removing all removable dominoes from $\lambda$ (this defines a unique partition). The number of dominoes removed from $\lambda$ is referred to as the weight of the partition $\lambda$ and is denoted $w(\lambda)$. Given $k, n \in \mathbb{N}_{0}$, we define $B_{k}(n)=\left\{\lambda \in \mathscr{P}_{n} \mid \operatorname{core}(\lambda)=\rho(k)\right\}$ to be the corresponding combinatorial 2-block. The set $\mathscr{P}_{n}$ decomposes as the disjoint union of the non-empty $B_{k}(n)$. We note that it makes sense to speak of the weight of a 2-block since any two partitions in the same 2-block necessarily have the same weight. Two simple $H_{-1}^{\mathbb{k}}(n)$-modules (or irreducible characters of $\mathbb{C S}_{n}$ ) belong to the same 2-block if and only if their labelling partitions belong to the same combinatorial 2-block (the same is true of Specht modules).

Example 1.5. The partition $\left(9,8,5,3^{2}, 2,1^{5}\right)$ has 4 removable dominoes: two (2)dominoes $\{(2,7),(2,8)\}$ and $\{(3,4),(3,5)\}$ and two $\left(1^{2}\right)$-dominoes $\{(4,3),(5,3)\}$ and $\{(10,1),(11,1)\}$. One can continue to successively remove such dominoes until one is left with the 2-core $\rho(5)=(5,4,3,2,1)$ as depicted on the left-hand-side of Figure 6.

Definition 1.6. Let $w_{1}, w_{2} \in \mathbb{N}_{0}$ be arbitrary and $\lambda \in \mathscr{P}_{w_{1}}$ and $\mu \in \mathscr{P}_{w_{2}}$ such that $\lambda_{1}^{T}+\mu_{1} \leqslant k+1$. We let $\tau_{\mu}^{\lambda}$ denote the partition

$$
\tau_{\mu}^{\lambda}=\left(\rho(k)+2 \mu^{T}\right)^{T}+2 \lambda .
$$



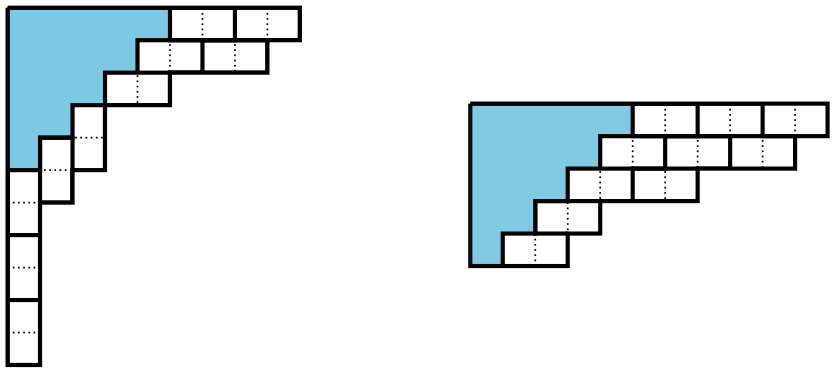

Figure 6. The partitions $\tau_{(3,1,1)}^{(2,2,1)}$ and $\tau_{\varnothing}^{\left(3^{2}, 2,1^{2}\right)}$ for $\rho=\rho(5)$

We say that any partition, $\tau_{\mu}^{\lambda}$, of this form is 2-separated.

Remark 1.7. We note that 2-separated partitions appear across all 2-blocks of the Hecke algebra. If the weight of a block is small compared to the size of the core, then all partitions in that block are 2-separated.

Remark 1.8. While the name "2-separated" may seem odd to some readers, it is motivated by the form this partition takes on a 2-abacus. In JM96] these partitions are referred to as "2-quotient separated".

1.4. Characters of 2-height zero. We now wish to discuss the defect groups of 2-blocks of symmetric groups and their characters of 2-height zero (see [JK] for background and more details). Write $n=2^{a_{1}}+\ldots+2^{a_{s}}$ where $a_{1}>\ldots>a_{s} \geqslant 0$; we set $s(n)=s$. For $m \in \mathbf{N}$, let $m_{2}$ be the largest 2-power dividing $m$. Then $(n !)_{2}=2^{n-s(n)}$ is the size of a Sylow 2-subgroup of $\mathfrak{S}_{n}$. Let $B$ be a 2-block of $\mathfrak{S}_{n}$ of weight $w$; then a defect group of $B$ is isomorphic to a Sylow 2-subgroup of $\mathfrak{S}_{2 w}$, and thus is of cardinality $2^{2 w-s(w)}$; the number $d(B)=2 w-s(w)$ is called the defect of $B$.

Example 1.9. The five 2-blocks of $\mathfrak{S}_{36}$ are indexed by the 2 -cores $\varnothing, \rho(3), \rho(4), \rho(7)$ and $\rho(8)$. These blocks are of weight $18,15,13,4$, and 0 respectively. Since $18=$ $2^{4}+2$, the 2 -block $B$ of weight 18 has defect $d(B)=34$.

We now recall the important notion of 2 -height 0 characters and simple modules. First we recall the fact that the dimension of any simple module $\mathbf{D}^{\mathbb{k}}(\lambda)$ or $\mathbf{S}^{\mathbb{C}}(\mu)$ belonging to a 2-block $B$ of the symmetric group $\mathfrak{S}_{n}$ is divisible by $2^{n-s(n)-d(B)}$. Such a module is said to be of 2-height 0 (or just height 0 if the prime $p=2$ is fixed in the context) if this 2-power is the largest 2-power dividing its dimension. We also say that the character $\chi^{\mu}$ associated to the Specht module $\mathbf{S}^{\mathbb{C}}(\mu)$ is a 2-height 0 character (or just height 0 character if the prime $p=2$ is fixed).

For the 2-block $B$, we set

$$
\operatorname{Irr}_{0}^{\mathbb{C}}(B)=\left\{\chi^{\lambda} \mid \chi^{\lambda} \text { is a height } 0 \text { character of } B\right\} .
$$

Generalising an earlier result of Macdonald on characters of odd degree, a combinatorial description for the partition labels of height 0 characters was given in terms of the so-called 2-core tower by Olsson (see 076, O93]). A new characterisation was recently given in GMT18, Section 3.2], again generalising an earlier version for the principal 2-block. This says that a partition $\lambda$ in a 2-block $B=B_{k}(n)$ of weight 
$w=2^{w_{1}}+\ldots+2^{w_{s(w)}}$, where $w_{1}>\ldots>w_{s(w)} \geq 0$, labels a height 0 character if and only if there is a sequence

$$
\lambda=\lambda(0) \supset \lambda(1) \supset \cdots \supset \lambda(s(w)-1) \supset \lambda(s(w))=\rho_{k}
$$

of partitions such that $\lambda(i-1) \backslash \lambda(i)$ is a $2^{w_{i}}$ rim-hook for $i=1, \ldots, s(w)$.

A formula for the number $k_{0}(B)$ of height 0 characters in a 2-block of weight $w$ was already given by Olsson [076], and was also deduced from the description above in GMT18. With $B$ and $w$ given as above, we have

$$
k_{0}(B)=\prod_{j=1}^{s(w)} 2^{w_{j}+1} .
$$

Since we get this number for each 2-block, the set of height 0 characters $\chi^{\mu}$ for $\mathfrak{S}_{n}$ constitutes quite a large class of irreducible characters.

Example 1.10. The irreducible characters of height 0 belonging to the principal 2-block of $\mathfrak{S}_{n}$ are precisely the characters of odd degree.

Example 1.11. The partitions $\left(9,8,3,2^{3}, 1^{2}\right),\left(9,6,5,2^{3}, 1^{2}\right) \vdash 28$ and the partitions $\left(9,6,5^{3}, 4,1^{2}\right)\left(10,7,4^{3}, 3,2^{2}\right) \vdash 36$ all label 2 -height zero characters. These partitions are depicted in Figure 2 in such a manner as to illustrate their combinatorial construction via adding rim hooks (detailed above).

Example 1.12. The five 2-blocks of $\mathfrak{S}_{36}$, their weights $w$, the 2-adic expansions of $2 w$, and the number of height 0 characters in the 2 -block are recorded in the table below.

\begin{tabular}{c|c|c|c|}
2 -core & weight $w$ & $2 w$ & $k_{0}(B)$ \\
\hline$\varnothing$ & 18 & $2^{5}+2^{2}$ & $2^{7}$ \\
$\rho(3)$ & 15 & $2^{4}+2^{3}+2^{2}+2$ & $2^{10}$ \\
$\rho(4)$ & 13 & $2^{4}+2^{3}+2$ & $2^{8}$ \\
$\rho(7)$ & 4 & $2^{3}$ & $2^{3}$ \\
$\rho(8)$ & 0 & - & 1
\end{tabular}

In particular, there are in total 1417 height 0 characters in 2-blocks of $\mathfrak{S}_{36}$, amongst which there are 128 of odd degree.

The following theorem will be one of the key results we use later on. It says that while there are many complex characters of height 0 , there is only one simple module $\mathbf{D}^{\mathbb{k}}(\lambda)$ of height 0 in each 2-block.

Theorem 1.13 ([KOW12, Theorem 1.4]). Let $\mathbb{k}$ be a field of characteristic 2. For any 2-block $B$ of weight $w$ of the symmetric group $\mathfrak{S}_{n}$, the module $\mathbf{D}^{\mathbb{k}}\left(\tau_{\varnothing}^{(w)}\right)$ is the unique simple $\mathbb{k}_{\mathfrak{S}_{n}}$-module in $B$ of height 0.

\section{KLR ALGEBRAS AND COLOURED TABLEAUX}

In this section, we assume $q$ is a primitive $e$ th root of unity. Given $n \in \mathbb{N}$ and an indeterminate $t$ we define the quantum integers and quantum factorials

$$
[n]_{t}=\frac{1+t^{2}+t^{4} \cdots+t^{2 n-2}}{t^{n-1}} \quad[n]_{t} !=[1]_{t}[2]_{t} \ldots[n]_{t}
$$

and given $\mu \in \mathscr{P}_{n}$ a partition of length $\ell$, we set

$$
[\mu]_{t} !=\left[\mu_{1}\right]_{t} !\left[\mu_{2}\right]_{t} ! \ldots\left[\mu_{\ell}\right]_{t} !
$$


We now define the quantum binomial coefficients to be

$$
\left[\begin{array}{l}
a \\
b
\end{array}\right]_{t}:=\frac{[a]_{t} !}{[b]_{t} ![a-b]_{t} !},
$$

for all $a \geqslant b \geqslant 0$. The motivating observation for studying Hecke algebras is the following. Let $\mathbb{k}$ be a field of characteristic $p$ and let $q \in \mathbb{k}$ be an element of order $e=p$ : then $H_{q}^{\mathrm{k}}(n)$ is isomorphic to $\mathbb{k}_{\mathfrak{s}}$. This gives us a way of factorising representation theoretic questions into two steps: firstly specialise the quantum parameter $q$ to be a $p$ th root of unity (in $\mathbb{C}$ and compatibly in $\mathbb{k}$ ) and study the nonsemisimple algebra $H_{q}^{\mathbb{C}}(n)$; then reduce modulo $p$ by studying $H_{q}^{\mathbb{k}}(n)=H_{q}^{\mathbb{Z}}(n) \otimes_{\mathbb{Z}} \mathbb{k}$. This allows us to factorise the problem of understanding decomposition matrices as follows,

$$
\left[\mathbf{S}_{q}^{\mathbb{k}}(\lambda): \mathbf{D}_{q}^{\mathbb{k}}(\mu)\right]=\left[\mathbf{S}_{q}^{\mathbb{C}}(\lambda): \mathbf{D}_{q}^{\mathbb{C}}(\nu)\right] \times\left[\mathbf{D}_{q}^{\mathbb{C}}(\nu) \otimes_{\mathbb{Z}} \mathbb{k}: \mathbf{D}_{q}^{\mathbb{k}}(\mu)\right] .
$$

On the right-hand side of the equality we have two matrices: the first is the decomposition matrix for $H_{q}^{\mathbb{C}}(n)$ and the second is known as "James' adjustment matrix". Therefore understanding the decomposition matrix of $H_{q}^{\mathbb{C}}(n)$ serves as a first step toward understanding the decomposition matrix of $\mathbb{k} \mathfrak{S}_{n}$.

We now recall the manner in which the grading can be incorporated into the picture and its immense power in understanding the decomposition matrix for $H_{q}^{\mathbb{C}}(n)$ (and hence, by equation (2.1) gives us a method for attacking the problem of calculating decomposition numbers for symmetric groups). Let $t$ be an indeterminate over $\mathbb{Z}$. The following theorem provides us with a $\mathbb{Z}$-graded presentation (which we record with respect to the indeterminate $t$ ) of the Hecke algebra.

Theorem 2.1 (BK09a, KL09, Rou08a $)$. The Hecke algebra $H_{q}^{\mathbb{k}}(n)$ admits a graded presentation with generators

$$
\left\{e(\underline{i}) \mid \underline{i}=\left(i_{1}, \ldots, i_{n}\right) \in(\mathbb{Z} / e \mathbb{Z})^{n}\right\} \cup\left\{y_{1}, \ldots, y_{n}\right\} \cup\left\{\psi_{1}, \ldots, \psi_{n-1}\right\},
$$

subject to a list of relations given in [BK09a, Main Theorem]. The $\mathbb{Z}$-grading on $H_{q}^{\mathbb{k}}(n)$ is given by

$$
\operatorname{deg}(e(\underline{i}))=0, \quad \operatorname{deg}\left(y_{r}\right)=2, \quad \operatorname{deg}\left(\psi_{r} e(\underline{i})\right)= \begin{cases}-2 & \text { if } i_{r}=i_{r+1}, \\ 1 \quad & \text { if } i_{r}=i_{r+1} \pm 1 \& e \neq 2, \\ 2 & \text { if } i_{r}=i_{r+1} \pm 1 \& e=2, \\ 0 & \text { otherwise }\end{cases}
$$

The importance of Theorem 2.1 is that it allows to consider an extra, richer graded structure on the Specht modules. We now recall the definition of this grading on the tableau basis of the Specht module. Let $\lambda \in \mathscr{P}_{n}$ and $\mathrm{t} \in \operatorname{Std}(\lambda)$. We let $\mathrm{t}^{-1}(k)$ denote the node in $\mathrm{t}$ containing the integer $k \in\{1, \ldots, n\}$. Given $1 \leqslant k \leqslant n$, we let $\mathcal{A}_{\mathrm{t}}(k)$, (respectively $\left.\mathcal{R}_{\mathrm{t}}(k)\right)$ denote the set of all addable res $\left(\mathrm{t}^{-1}(k)\right)$-nodes (respectively all removable res $\left(\mathrm{t}^{-1}(k)\right)$-nodes) of the partition Shape $\left(\mathrm{t}_{\downarrow}{ }_{\{1, \ldots, k\}}\right)$ which are above $\mathrm{t}^{-1}(k)$, i.e. those in an earlier row. Let $\lambda \in \mathscr{P}_{n}$ and $\mathrm{t} \in \operatorname{Std}(\lambda)$. We define the degree of $\mathrm{t}$ as follows:

$$
\operatorname{deg}(\mathrm{t})=\sum_{k=1}^{n}\left(\left|\mathcal{A}_{\mathrm{t}}(k)\right|-\left|\mathcal{R}_{\mathrm{t}}(k)\right|\right) .
$$

Given $\mathrm{t} \in \operatorname{Std}(\lambda)$ we define the residue sequence of $\mathrm{t}$ as follows:

$$
\operatorname{res}(\mathrm{t})=\left(\operatorname{res}\left(\mathrm{t}^{-1}(1)\right), \operatorname{res}\left(\mathrm{t}^{-1}(2)\right), \ldots, \operatorname{res}\left(\mathrm{t}^{-1}(n)\right)\right) \in(\mathbb{Z} / e \mathbb{Z})^{n}
$$


and we write $e(\mathrm{t}):=e(\operatorname{res}(\mathrm{t})) \in H_{q}^{\mathbb{k}}(n)$. Let $t$ be an indeterminate over $\mathbb{N}_{0}$. If $M=\oplus_{z \in \mathbb{Z}} M_{z}$ is a free graded $\mathbb{k}$-module, then its graded dimension is the Laurent polynomial

$$
\operatorname{Dim}_{t}(M)=\sum_{k \in \mathbb{Z}}\left(\operatorname{dim}_{\mathbb{k}} M_{k}\right) t^{k}
$$

If $M$ is a graded $H_{q}^{\mathbb{k}}(n)$-module and $k \in \mathbb{Z}$, define $M\langle k\rangle$ to be the same module with $(M\langle k\rangle)_{i}=M_{i-k}$ for all $i \in \mathbb{Z}$. We call this a degree shift by $k$. The graded dimensions of Specht modules admit a combinatorial description as follows:

Theorem 2.2 ([BKW11]). The Specht module $\mathbf{S}_{q}^{\mathrm{k}}(\lambda)$ is a free $\mathbb{Z}$-graded $\mathbb{k}$-module with basis $\left\{e(\mathrm{t}) \psi^{\mathrm{t}} \mid \mathrm{t} \in \operatorname{Std}(\lambda)\right\}$ and where $\operatorname{deg}\left(\psi^{\mathrm{t}}\right)=t^{\operatorname{deg}(\mathrm{t})}$.

Of course, this theorem gives us an added level of graded structure to consider: the graded decomposition numbers of symmetric groups and their Hecke algebras. By Theorem 2.2 we obtain a grading on the module $\mathbf{D}_{q}(\mu)=\mathbf{S}_{q}(\lambda) / \operatorname{rad}\left(\mathbf{S}_{q}(\lambda)\right)$. We define the graded decomposition number to be the polynomial

$$
d_{\lambda, \mu}^{\mathbb{k}}(t)=\sum_{k \in \mathbb{Z}}\left[\mathbf{S}_{q}^{\mathbb{k}}(\lambda): \mathbf{D}_{q}^{\mathbb{k}}(\mu)\langle k\rangle\right] t^{k}
$$

which records the composition multiplicity of each simple module and its relevant degree shift. In particular upon specialisation $t \rightarrow 1$ the polynomials of equation (2.3) specialise to be the usual decomposition numbers. While one might expect this grading to increase the level of difficulty of our question, we find that by keeping track of this extra grading information we are rewarded with an incredibly powerful algorithm for understanding the decomposition numbers of $H_{q}^{\mathbb{C}}(n)$.

Equation (2.1) hints that we could first study the decomposition numbers of $H_{q}^{\mathbb{C}}(n)$ as an intermediary first step toward understanding the decomposition numbers of symmetric groups in positive characteristic. In fact, this approach has been incredibly successful: Lascoux, Leclerc and Thibon provided an iterative algorithm for understanding the graded decomposition numbers of $H_{q}^{\mathbb{C}}(n)$ in [LLT96. We now provide an elementary tableau-theoretic re-interpretation of this algorithm (using the work of Kleshchev and Nash [KN10]).

2.1. Coloured tableaux. We now recast ideas from KN10] in terms of orbits of tableaux which we encode as "coloured tableaux". This "colouring" comes from the observation that each idempotent truncation of a Specht module, $e(\underline{i}) \mathbf{S}_{q}(\mu)$, has a homogeneous basis indexed by the $\mathrm{t} \in \operatorname{Std}(\lambda) \operatorname{such}$ that $\operatorname{res}(\mathrm{t})=\underline{i} \in(\mathbb{Z} / e \mathbb{Z})^{n}$, by Theorem 2.2. We now develop these ideas further.

Let $\lambda$ a partition of $n$ and $\mu$ a composition of $n$. We define a Young tableau of shape $\lambda$ and weight $\mu$ to be a filling of the nodes of $\lambda$ with the entries

$$
\underbrace{1, \ldots, 1}_{\mu_{1}}, \underbrace{2, \ldots, 2}_{\mu_{2}}, \ldots, \underbrace{\ell, \ldots, \ell}_{\mu_{\ell}} .
$$

We say that a tableau is row standard if the entries are weakly increasing along the rows of $\lambda$; we denote the set of such tableaux by $\operatorname{RStd}(\lambda, \mu)$. We say that the Young tableau is semistandard if the entries are weakly increasing along the rows and are strictly increasing along the columns of $\lambda$; we denote the set of such tableaux by $\operatorname{SStd}(\lambda, \mu)$. 
Definition 2.3. Given $\mu \vdash_{e} n$, we let $\operatorname{Lad}(\mu)$ denote the composition $\nu$ such that

$$
\nu_{i}=\sharp\{(r, c) \in \mu \mid \mathfrak{l}(r, c)=i\},
$$

where we have that $\nu_{1}=0$ by definition. We define a semistandard coloured tableaux, S, to be a semistandard tableau of weight $\operatorname{Lad}(\mu)$ such that the entry of any node is congruent to its residue. We denote the set of all such tableaux of shape $\lambda$ by $\operatorname{CStd}(\lambda, \mu) \subseteq \operatorname{SStd}(\lambda, \operatorname{Lad}(\mu))$. We let $\mathrm{L}^{\mu}$ denote the unique element of $\operatorname{CStd}(\mu, \mu)$. We set $e(\mu)=e\left(\operatorname{res}\left(\mathrm{L}^{\mu}\right)\right) \in H_{q}^{\mathbb{k}}(n)$.

Example 2.4. For $q=-1, e=2$ and $\mu=(6),(5,1),(4,2)$, we have that $\operatorname{Lad}(\mu)$ is equal to $(0,1,1,1,1,1,1),(0,1,2,1,1,1)$ and $(0,1,2,2,1)$ respectively. All semistandard coloured tableaux (up to conjugation) for the principal 2-block of $H_{-1}^{\mathbb{k}}(6)$ are listed in the table below.

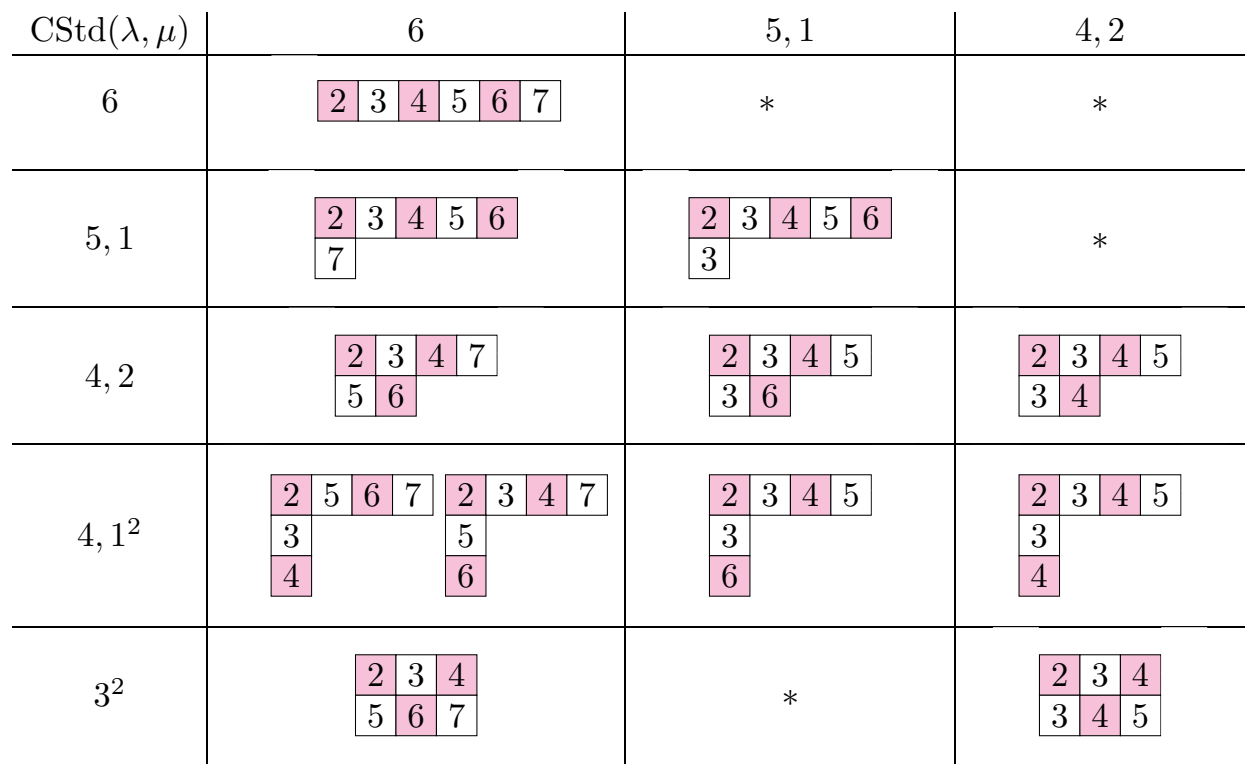

The importance of coloured semistandard tableaux of weight $\mu$ is that they encode an $\mathfrak{S}_{\operatorname{Lad}(\mu)}$-orbit of standard Young tableaux; we shall now make this idea more precise. Given a composition $\nu$ and $c \geqslant 1$, we set $[\nu]_{c}=\nu_{1}+\nu_{2}+\cdots+\nu_{c} \in \mathbb{N}$. Let $\mu$ be an $e$-regular partition and let $\mathrm{s}$ be a standard Young tableau of shape $\lambda$ such that the residue sequence of $s$ is given by

$$
0, \underbrace{-1,-1, \ldots,-1}_{\nu_{e+1} \text { times }}, \underbrace{-2,-2, \ldots,-2}_{\nu_{e+2} \text { times }}, \underbrace{-3,-3, \ldots,-3}_{\nu_{e+3} \text { times }}, \ldots
$$

for $\nu=\left(0, \ldots, 0,1, \nu_{e+1}, \ldots, \nu_{\ell}\right)=\operatorname{Lad}(\mu)$; we refer to such an $\mathrm{s}$ as a ladder tableau of ladder weight $\mu$. Then define $\mu(\mathrm{s})$ to be the coloured tableau obtained from $\mathrm{s}$ by replacing each entry $i$ for $[\operatorname{Lad}(\mu)]_{c-1}<i \leqslant[\operatorname{Lad}(\mu)]_{c}$ in s by the entry $c$ for $c \geqslant 1$.

We identify a coloured semistandard Young tableau, S, of weight $\mu$ with the set of standard Young tableaux, $[\mathrm{S}]_{\mu}=\{\mathrm{p} \mid \mu(\mathrm{p})=\mathrm{S}\}$. Given $\mathrm{S} \in \operatorname{SStd}(\lambda, \mu)$ we let $\mathrm{p}^{\lambda} \in[\mathrm{S}]_{\mu}$ denote the unique most dominant tableau in $[S]_{\mu}$. 
Example 2.5. Continuing with Example 2.4, we let $\mathrm{S} \in \operatorname{CStd}\left(\left(4,1^{2}\right),(4,2)\right)$ depicted above. We have that

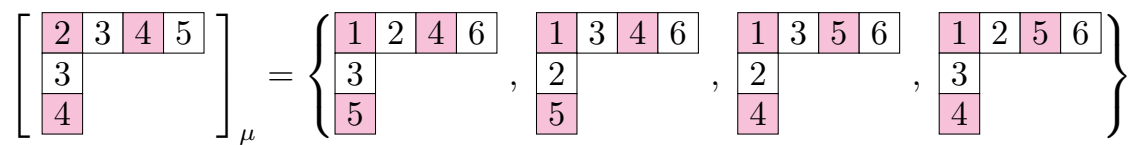

as an orbit of standard tableaux (which we have coloured in order to facilitate comparison).

For $\mu \in \mathscr{R}_{n}^{e}$, we let $\mathrm{L}^{\mu}$ be the unique element of $\operatorname{CStd}(\mu, \mu)$.

Proposition 2.6. We have that

$$
\operatorname{Dim}_{t}\left(e(\mu) \mathbf{S}_{q}(\mu)\right)=\operatorname{Dim}_{t}\left(e(\mu) \mathbf{D}_{q}(\mu)\right)=\sum_{\mathbf{t} \in \mathrm{L}^{\mu}} t^{\operatorname{deg}(\mathrm{t})}=[\operatorname{Lad}(\mu)]_{t} !
$$

Proof. We have that $e(\mu) \mathbf{S}_{q}(\lambda)=0$ for any $\lambda \triangleright \mu$ (by definition of the ladder tableau) and so the first and second equalities hold by Theorem 1.4 and the definition of the ladder tableau as an orbit. The final equality is not difficult, but is explicitly proven in KN10, Lemma 3.4].

By the above, the orbit sum $\sum_{\mathrm{t} \in \mathrm{L}^{\mu}} t^{\mathrm{deg}(\mathrm{t})}$ is invariant under the bar map interchanging $t \leftrightarrow t^{-1}$ and so we keep track of this by formally setting $\operatorname{deg}\left(\mathrm{L}^{\mu}\right)=0$. We now provide a general definition of the degree of a coloured tableau which allows us to calculate the graded characters of weight spaces of Specht modules in terms of coloured tableaux. Let $(a, b) \in \lambda \in \mathscr{P}_{n}$ be a node of residue $i \in \mathbb{Z} / e \mathbb{Z}$ and $\mu \in \mathscr{R}_{n}^{e}, \mathrm{~S} \in \operatorname{CStd}(\lambda, \mu)$. We let $\mathcal{A}_{\mathrm{S}}(a, b)$ denote the set of all addable $i$-nodes of the partition

$$
\lambda \cap\{(r, c) \mid \mathrm{S}(r, c) \leqslant \mathfrak{l}(a, b)\}
$$

which are above $(a, b) \in \lambda$. We let $\mathcal{R}_{\mathrm{S}}(a, b)$ denote the set of all removable $i$-nodes of the partition

$$
\lambda \cap\{(r, c) \mid \mathrm{S}(r, c)<\mathfrak{l}(a, b)\}
$$

which are above $(a, b) \in \lambda$. We then define the degree of the node $(a, b) \in \lambda$ to be $\left|\mathcal{A}_{\mathrm{S}}(a, b)\right|-\left|\mathcal{R}_{\mathrm{S}}(a, b)\right|$. We define $\operatorname{deg}(\mathrm{S})$ to be the sum over the degrees of all nodes $(a, b) \in \lambda$.

We have seen that the tableaux of $\operatorname{CStd}(\lambda, \mu)$ are simply the orbits of tableaux from $\operatorname{Std}(\lambda)$ with a given residue sequence. Therefore, by comparing the degree function for coloured tableaux with that of standard tableaux we obtain

$$
\operatorname{Dim}_{t}\left(e(\mu) \mathbf{S}_{q}(\lambda)\right)=[\operatorname{Lad}(\mu)]_{t} ! \sum_{\mathbf{S} \in \operatorname{CStd}(\lambda, \mu)} t^{\operatorname{deg}(\mathrm{S})} .
$$

And so coloured standard tableaux provide a combinatorial description of the ladder-weight multiplicity as defined in [KN10, Section 3.3].

Example 2.7. Continuing with $\mathrm{S} \in \operatorname{CStd}\left(\left(4,1^{2}\right),(4,2)\right)$ in Example 2.5 we have that

$$
\mathcal{R}_{\mathrm{S}}(a, b)=\emptyset \text { for all }(a, b) \in\left(4,1^{2}\right) \quad \text { and } \quad \mathcal{A}_{\mathrm{S}}(a, b)= \begin{cases}\{(2,2)\} & \text { if }(a, b)=(3,1), \\ \emptyset & \text { otherwise }\end{cases}
$$

and therefore

$$
\operatorname{deg}_{\mathrm{S}}(a, b)= \begin{cases}1 & \text { if }(a, b)=(3,1) \\ 0 & \text { otherwise }\end{cases}
$$


Therefore $\operatorname{deg}(S)=1$. The four distinct standard tableaux $\mathbf{s} \in[S]_{(4,2)}$ are depicted in Example 2.5, these four tableaux are obtained from each other by permuting the pairs 2,3 in the third ladder and the pairs 4,5 in the fourth ladder. We have that $\sum_{\mathbf{s} \in \mathrm{S}} t^{\operatorname{deg}(\mathbf{s})}=t^{3}+2 t+t^{-1}=t \times\left(t+t^{-1}\right)^{2}=\operatorname{deg}(\mathrm{S}) \times[2]_{t} ![2]_{t} !=\operatorname{deg}(\mathrm{S}) \times[\operatorname{Lad}(\mu)]_{t} !$.

With our new tableaux theoretic combinatorics in place, we can recast the (LLT) algorithm from [KN10, Section 4] in this combinatorial setting.

Example 2.8. We record the graded degrees of the coloured tableaux appearing in Example 2.4 and their conjugates (which are not pictured). Notice that conjugation does not preserve the degrees of tableaux.

\begin{tabular}{c|ccc} 
& 6 & 5,1 & 4,2 \\
\hline 6 & 1 & $*$ & $*$ \\
5,1 & $t$ & 1 & $*$ \\
4,2 & 1 & $t$ & 1 \\
$4,1^{2}$ & $2 t$ & $t^{2}$ & $t$ \\
$3^{2}$ & $t$ & $*$ & $t$ \\
$2^{3}$ & $t^{2}$ & $*$ & $t^{2}$ \\
$3,1^{3}$ & $2 t^{2}$ & $t$ & $t^{2}$ \\
$2^{2}, 1^{2}$ & $t^{3}$ & $t^{2}$ & $t^{3}$ \\
$2,1^{4}$ & $t^{2}$ & $t^{3}$ & $*$ \\
$1^{6}$ & $t^{3}$ & $*$ & $*$
\end{tabular}

We are almost ready to restate the LLT algorithm in terms of our combinatorics, we simply require two observations about the graded structure of the Hecke algebra. The first is almost trivial, but the proof of the latter depends on incredibly deep geometric or categorical insights.

Theorem 2.9 ([BK09b, Theorem 4.18]). For $\lambda \in \mathscr{P}_{n}$ and $\mu \in \mathscr{R}_{n}^{e}$, the polynomial $\operatorname{Dim}_{t}\left(e(\mu) \mathbf{D}_{q}^{\mathbb{k}}(\lambda)\right)$ is bar-invariant (i.e., fixed under interchanging $t$ and $t^{-1}$ ).

Theorem 2.10 (VV99). Let $\mathbb{k}=\mathbb{C}$. For $\lambda \in \mathscr{P}_{n}$ and $\mu \in \mathscr{R}_{n}^{e}$ with $\mu \neq \lambda$, $d_{\lambda, \mu}(t) \in t \mathbb{N}_{0}[t]$.

Rearranging [KN10, Theorem 3.8] in terms of our coloured tableaux, we obtain the following relationships between coloured tableaux, simple characters, and graded decomposition numbers:

Proposition 2.11. For $\lambda \in \mathscr{P}_{n}$ and $\mu \in \mathscr{R}_{n}^{e}$ we have that

$\operatorname{Dim}_{t}\left(e(\mu) \mathbf{S}^{\mathbb{k}}(\lambda)\right)$

$$
=\sum_{\mathrm{S} \in \operatorname{CStd}(\lambda, \mu)} t^{\operatorname{deg}(\mathrm{S})}[\operatorname{Lad}(\mu)]_{t} ! \in \mathbb{N}_{0}\left[t, t^{-1}\right] \quad \text { and } \quad \operatorname{Dim}_{t}\left(e(\mu) \mathbf{D}_{q}^{\mathbb{k}}(\lambda)\right) \in \mathbb{N}_{0}\left[t+t^{-1}\right] .
$$

Moreover, the following hold:

(i) if $\operatorname{CStd}(\lambda, \mu)=\emptyset$, then $d_{\lambda, \mu}(t)=0$ and $\operatorname{Dim}_{t}\left(e(\mu) \mathbf{D}_{q}^{\mathbb{k}}(\lambda)\right)=0$;

(ii) we have $\left.\operatorname{Dim}_{t}\left(e(\mu) \mathbf{S}_{q}^{\mathbb{k}}(\mu)\right)\right)=\operatorname{Dim}_{t}\left(e(\mu) \mathbf{D}_{q}^{\mathbb{k}}(\mu)\right)=[\operatorname{Lad}(\mu)]_{t}$ !;

(iii) we have that, for $\lambda \in \mathscr{R}_{n}^{e}$,

$$
\begin{aligned}
& \operatorname{Dim}_{t}\left(e(\mu) \mathbf{D}_{q}^{\mathbb{k}}(\lambda)\right)+d_{\lambda, \mu}(t)[\operatorname{Lad}(\mu)]_{t} ! \\
& \quad=\sum_{\mathrm{S} \in \operatorname{CStd}(\lambda, \mu)} t^{\operatorname{deg}(\mathrm{S})}[\operatorname{Lad}(\mu)]_{t} !-\sum_{\lambda \triangleleft \nu \triangleleft \mu} \operatorname{Dim}_{t}\left(e(\mu) \mathbf{D}_{q}^{\mathbb{k}}(\nu)\right) d_{\lambda, \nu}(t) .
\end{aligned}
$$


Now we set $\mathbb{k}=\mathbb{C}$. The right-hand side of the equation in Proposition 2.11 ( $i i i)$ is calculated by induction along the dominance ordering. Any polynomial in $\mathbb{N}_{0}\left[t, t^{-1}\right]$ can be written uniquely as the sum of a bar-invariant polynomial from $\mathbb{N}_{0}\left[t, t^{-1}\right]$ and a polynomial from $t \mathbb{N}_{0}[t]$. Putting together Theorems 2.9 and 2.10 we deduce that the left-hand-side is uniquely determined by the right-hand-side and induction on the dominance order.

Example 2.12. We continue with Example 2.4. Using the equation in Proposition 2.11 (iii), we obtain the first 5 rows of the graded decomposition matrix of the principal block of $H_{-1}^{\mathbb{C}}(6)$ and $\frac{1}{[\operatorname{Lad}(\mu)]_{t} !} \operatorname{Dim}_{t}\left(e(\mu) \mathbf{D}_{q}(\lambda)\right)$ as follows:

\begin{tabular}{c|ccc} 
& 6 & 5,1 & 4,2 \\
\hline 6 & 1 & $*$ & $*$ \\
5,1 & $t$ & 1 & $*$ \\
4,2 & $*$ & $t$ & 1 \\
$4,1^{2}$ & $t$ & $t^{2}$ & $t$ \\
$3^{2}$ & $*$ & $*$ & $t$ \\
$2^{3}$ & $*$ & $*$ & $t^{2}$ \\
$3,1^{3}$ & $t^{2}$ & $t$ & $t^{2}$ \\
$2^{2}, 1^{2}$ & $*$ & $t^{2}$ & $t^{3}$ \\
$2,1^{4}$ & $t^{2}$ & $t^{3}$ & $*$ \\
$1^{6}$ & $t^{3}$ & $*$ & $*$
\end{tabular}

\begin{tabular}{c|ccc} 
& 6 & 5,1 & 4,2 \\
\hline 6 & 1 & $*$ & $*$ \\
5,1 & $*$ & 1 & $*$ \\
4,2 & 1 & $*$ & 1
\end{tabular}

Notice that if we multiply these two matrices together we obtain the matrix from Example 2.8. The remaining entries of the table can be deduced by applying the sign automorphism to the Specht modules (although this automorphism is not of degree zero and so the entries will differ by a degree shift). Comparing with the table in Example 2.8, we observe that the entry in the row labelled by $(4,2)$ and column labelled by (6) is bar-invariant in Example 2.8 and so does not contribute to the decomposition matrix, but instead contributes a vector in the simple module $\mathbf{D}_{-1}(4,2)$. Then in the row labelled by $\left(4,1^{2}\right)$ we see another discrepancy between the two tables: this is because $\mathbf{D}_{-1}(4,2)$ is a composition factor of $\mathbf{S}_{-1}\left(4,1^{2}\right)$ and so it contributes to the sum in Proposition 2.11( $($ iii).

We are now ready to provide new upper bounds for (graded) decomposition numbers in terms of our coloured tableaux.

Theorem 2.13. For $\lambda \in \mathscr{P}_{n}$ and $\mu \in \mathcal{R}_{n}^{e}$ and $\mathbb{k}$ an arbitrary field, we have that

$$
\left[\mathbf{S}_{q}^{\mathbb{k}}(\lambda): \mathbf{D}_{q}^{\mathbb{k}}(\mu)\langle k\rangle\right] \leqslant|\{\mathrm{S} \mid \mathrm{S} \in \operatorname{CStd}(\lambda, \mu), \operatorname{deg}(\mathrm{S})=k\}|
$$

for $k \in \mathbb{Z}$ and in particular, $d_{\lambda, \mu}^{\mathbb{k}} \leqslant|\operatorname{CStd}(\lambda, \mu)|$.

Proof. It is immediate from equation (2.4) that

$$
\left[\mathbf{S}_{q}^{\mathbb{k}}(\lambda): \mathbf{D}_{q}^{\mathbb{k}}(\mu)\langle k\rangle\right] \leqslant[\operatorname{Lad}(\mu)]_{t} ! \times|\{\mathrm{S} \mid \mathrm{S} \in \operatorname{CStd}(\lambda, \mu), \operatorname{deg}(\mathrm{S})=k\}|
$$

and indeed this is just rephrasing a classical observation due to Gordon James. The new observation is that by Proposition 2.11, we know that $[\operatorname{Lad}(\mu)]_{t}$ ! divides both

$$
\operatorname{Dim}_{t}\left(e(\mu)\left(\mathbf{S}_{q}^{\mathbb{k}}(\lambda)\right) \quad \operatorname{Dim}_{t}\left(e(\mu)\left(\mathbf{D}_{q}^{\mathbb{k}}(\mu)\right)\right)\right.
$$

and the result follows by induction on the dominance ordering and the equation in Proposition 2.11 ( $i i i)$. In more detail, our base case for induction is when $\mu=\lambda$ mentioned above. Now, by Proposition 2.11( 
result holds for all $\nu$ such that $\mu \triangleright \nu \triangleright \lambda$. Putting this together with Proposition $2.11(i i)$, we deduce that $[\operatorname{Lad}(\mu)]_{t}$ ! divides $\operatorname{Dim}_{t}\left(e(\mu) \mathbf{D}_{q}^{\mathbb{k}}(\lambda)\right)$ as required.

Example 2.14. If $p=2$ then the graded decomposition matrix of $\mathbb{k} \mathfrak{S}_{6}$ is given by the table in Example 2.8, In other words, the bounds of Theorem 2.13 are sharp.

Remark 2.15. The inductive approach to calculating decomposition numbers of $H_{q}^{\mathbb{C}}(n)$ highlighted in the equation in Proposition 2.11 $($ iii $)$ above is used in the arXiv appendix to this paper to prove decomposability of an infinite family of Specht modules. In Section 4 the above algorithm will not work (as the set of 2separated partitions is not saturated in the dominance order). However, we provide an analogous algorithm for calculating 2-separated decomposition numbers using "2-dilated" coloured tableaux.

\section{The Cherednik algebra and a simple Criterion for semisimplicity of A Specht MOdule}

The group $\mathfrak{S}_{n}$ acts on the algebra, $\mathbb{C}\left\langle x_{1}, \ldots, x_{n}, y_{1}, \ldots y_{n}\right\rangle$, of polynomials in $2 n$ non-commuting variables. The rational Cherednik algebra $\mathscr{H}_{q}\left(\mathfrak{S}_{n}\right)$ is a quotient of the semidirect product algebra $\mathbb{C}\left\langle x_{1}, \ldots, x_{n}, y_{1}, \ldots, y_{n}\right\rangle \rtimes \mathfrak{S}_{n}$ by commutation relations in the $x$ 's and $y$ 's that are similar to those of the Weyl algebra but involve an error term in $\mathbb{C S}_{n}$ (see [EG02, Section 1] for the full list of relations). In particular, these relations tell us that the $x$ 's commute with each other and so do the $y$ 's. The algebra $\mathscr{H}_{q}\left(\mathfrak{S}_{n}\right)$ has three distinguished subalgebras: $\mathbb{C}[\underline{y}]:=$ $\mathbb{C}\left[y_{1}, \ldots, y_{n}\right], \mathbb{C}[\underline{x}]:=\mathbb{C}\left[x_{1}, \ldots, x_{n}\right]$, and the group algebra $\mathbb{C S}_{n}$. The $P B W$ theorem EG02, Theorem 1.3] asserts that multiplication gives a vector space isomorphism

$$
\mathbb{C}[\underline{x}] \otimes \mathbb{C S}_{n} \otimes \mathbb{C}[\underline{y}] \stackrel{\cong}{\longrightarrow} \mathscr{H}_{q}\left(\mathfrak{S}_{n}\right)
$$

called the triangular decomposition of $\mathscr{H}_{q}\left(\mathfrak{S}_{n}\right)$, by analogy with the triangular decomposition of the universal enveloping algebra of a semisimple Lie algebra. We define the category $\mathcal{O}_{q}\left(\mathfrak{S}_{n}\right)$ to be the full subcategory consisting of all finitely generated $\mathscr{H}_{q}\left(\mathfrak{S}_{n}\right)$-modules on which $y_{1}, \ldots, y_{n}$ act locally nilpotently. The category $\mathcal{O}_{q}\left(\mathfrak{S}_{n}\right)$ is a highest weight category with respect to the poset $\left(\mathscr{P}_{n}, \triangleright\right)$. The standard modules are constructed as follows. Extend the action of $\mathfrak{S}_{n}$ on $\mathbf{S}^{\mathbb{C}}(\lambda)$ to an action of $\mathbb{C}[\underline{y}] \rtimes \mathfrak{S}_{n}$ by letting $y_{1}, \ldots, y_{n}$ act by 0 . The algebra $\mathbb{C}[\underline{y}] \rtimes \mathfrak{S}_{n}$ is a subalgebra of $\overline{\mathscr{H}}_{q}\left(\mathfrak{S}_{n}\right)$ and we define the Weyl modules,

$$
\Delta(\lambda):=\operatorname{Ind}_{\mathbb{C}[\underline{y}] \rtimes \mathfrak{S}_{n}}^{\mathscr{H}_{q}\left(\mathfrak{S}_{n}\right)} \mathbf{S}^{\mathbb{C}}(\lambda):=\mathscr{H}_{q}\left(\mathfrak{S}_{n}\right) \otimes_{\mathbb{C}[\underline{y}] \rtimes \mathfrak{S}_{n}} \mathbf{S}^{\mathbb{C}}(\lambda)=\mathbb{C}[\underline{x}] \otimes \mathbf{S}^{\mathbb{C}}(\lambda),
$$

where the last equality is only as $\mathbb{C}[\underline{x}]$-modules and follows from the triangular decomposition. We let $L(\lambda)$ denote the unique irreducible quotient of $\Delta(\lambda)$. In [RSVV16, Theorem 7.4.] (see also Los16, Web13]) it is shown that $\mathcal{O}_{q}\left(\mathfrak{S}_{n}\right)$ is standard Koszul. We do not recall the definition of a standard Koszul algebra here, but merely the following useful proposition. The following proposition is proven in [BGS96, Proposition 2.4.1] in the generality of all Koszul algebras.

Proposition 3.1. For $\lambda, \mu \in \mathscr{P}_{n}$ we have that

$$
[\Delta(\lambda): L(\mu)\langle i\rangle]=\operatorname{dim}_{\mathbb{k}} \operatorname{Hom}_{\mathscr{H}_{q}\left(\mathfrak{S}_{n}\right)}\left(\operatorname{rad}_{i}\left(\Delta^{\mathbb{C}}(\lambda)\right), L^{\mathbb{C}}(\mu)\right) .
$$

Proof. By [BGS96, Corollary 2.3.3], any Koszul algebra is quadratic. Therefore, since $\Delta(\lambda) / \operatorname{rad}(\Delta(\lambda))=L(\lambda)$ is simple and concentrated in degree zero, the radical 
filtration of $\Delta(\lambda)$ coincides with the grading filtration of $\Delta(\lambda)$ by [BGS96, Proposition 2.4.1].

Now, there exists an exact functor (the Knizhnik-Zamolodchikov functor) relating the module categories of Cherednik and Hecke algebras,

$$
\mathrm{KZ}: \mathcal{O}_{q}\left(\mathfrak{S}_{n}\right) \longrightarrow H_{q}^{\mathbb{C}}(n)-\bmod
$$

A construction of this functor is given in GGOR03, here we will only need the fact (from [GGOR03, Section 6]) that

$$
\mathrm{KZ}(\Delta(\lambda))=\mathbf{S}_{q}^{\mathbb{C}}(\lambda), \quad \mathrm{KZ}(L(\lambda))= \begin{cases}\mathbf{D}_{q}^{\mathbb{C}}(\lambda) & \text { if } \lambda \text { is } e \text {-regular } \\ 0 & \text { otherwise }\end{cases}
$$

This allows us to prove the following criterion for decomposability of Specht modules, denoted $\mathbf{S}_{q}^{\mathbb{C}}(\lambda)$, for the Hecke algebra $H_{q}^{\mathbb{C}}(n)$.

Theorem 3.2. Fix $\lambda \in \mathscr{P}_{n}$. Suppose that for all e-regular partitions $\mu$, we have that

$$
\left[\mathbf{S}_{q}^{\mathbb{C}}(\lambda): \mathbf{D}_{q}^{\mathbb{C}}(\mu)\right]=a_{\mu} t^{p(\lambda)}
$$

for some fixed $p(\lambda)=z \in \mathbb{N}$ (independent of $\mu$ ) and some scalars $a_{\mu} \in \mathbb{N}$. It follows that the Specht module $\mathbf{S}_{q}^{\mathbb{C}}(\lambda)$ is semisimple.

Proof. Throughout the proof, we let $\lambda \in \mathscr{P}_{n}$ be an arbitrary partition. For an $e$-regular partition $\mu \in \mathscr{P}_{n}$, we have that

$$
[\Delta(\lambda): L(\mu)\langle k\rangle]=[\mathrm{KZ}(\Delta(\lambda)): \mathrm{KZ}(L(\mu)\langle k\rangle)]=\left[\mathbf{S}_{q}^{\mathbb{C}}(\lambda): \mathbf{D}_{q}^{\mathbb{C}}(\mu)\langle k\rangle\right]
$$

by equation (3.1). Putting together Proposition 3.1 and our assumption in equation (3.2), we have that

$$
\left[\operatorname{rad}_{i}(\Delta(\lambda)): L(\mu)\right]=0
$$

for $\mu$ any $e$-regular partition and any $i \neq z$. Therefore

$$
\mathrm{KZ}\left(\operatorname{rad}_{i}(\Delta(\lambda))= \begin{cases}\bigoplus_{\mu} a_{\mu} \mathbf{D}_{q}^{\mathbb{C}}(\mu)\langle z\rangle & \text { for } i=z, \\ 0 & \text { otherwise. }\end{cases}\right.
$$

Therefore

$$
\mathrm{KZ}(\Delta(\lambda))=\mathrm{KZ}\left(\operatorname{rad}_{z}(\Delta(\lambda))\right)=\bigoplus_{\mu} a_{\mu} \mathbf{D}_{q}^{\mathbb{C}}(\mu)\langle z\rangle
$$

and the result follows.

Remark 3.3. We have seen the grading and radical structure of standard $\mathcal{O}_{q}\left(\mathfrak{S}_{n}\right)$ modules are intimately related. It is unknown as to whether or not the Schur functor preserves this property. Thus Theorem 3.2 represents all that is currently known about the relationship between the grading and radical structure on Specht modules for $H_{q}^{\mathbb{C}}(n)$.

Remark 3.4. For the reader unfamiliar with Cherednik algebras, one can also deduce the results of this section using the language of quiver Schur algebras and appealing to SW11. 


\section{The Hecke algebra and 2-Separated partitions}

Throughout this section, we shall consider the representation theory of the Hecke algebra $H_{-1}^{\mathbb{C}}(n)$ as a first approximation to the 2-modular representation theory of symmetric groups. We focus on the Specht modules labelled by 2 -separated partitions. We shall prove that these modules are semisimple and decompose them as a direct sum of graded simple modules.

Theorem 4.1. We set $\tau:=\tau(k)$. We have that

$$
\operatorname{Dim}_{t}\left(e\left(\tau_{\varnothing}^{\alpha}\right) \mathbf{S}_{-1}^{\mathbb{C}}\left(\tau_{\mu}^{\lambda}\right)\right)=\left|\operatorname{SStd}\left(\lambda^{T} \cup \mu, \alpha^{T}\right)\right| \times\left[\alpha^{T}\right]_{t} !^{2} \times[k]_{t} ! \times t^{|\mu|} .
$$

Before embarking on the proof, we note the following immediate corollaries.

Corollary 4.2. We have that

$$
\operatorname{Dim}_{t}\left(e\left(\tau_{\varnothing}^{\alpha}\right) \mathbf{S}_{-1}^{\mathbb{C}}\left(\tau_{\mu}^{\lambda}\right)\right)=t^{|\mu|} \sum_{\nu} c\left(\nu^{T}, \lambda^{T}, \mu\right) \operatorname{Dim}_{t}\left(e\left(\tau_{\varnothing}^{\alpha}\right) \mathbf{D}_{-1}^{\mathbb{C}}\left(\tau_{\varnothing}^{\nu}\right)\right) .
$$

Therefore, as an $H_{-1}^{\mathbb{C}}(n)$-module, any Specht module labelled by a 2-separated partition is semisimple and decomposes as follows

$$
\mathbf{S}_{-1}^{\mathbb{C}}\left(\tau_{\mu}^{\lambda}\right)=\bigoplus_{\nu} c\left(\nu^{T}, \lambda^{T}, \mu\right) \mathbf{D}_{-1}^{\mathbb{C}}\left(\tau_{\varnothing}^{\nu}\right)\langle|\mu|\rangle .
$$

In particular, the Specht $H_{-1}^{\mathbb{C}}(n)$-module $\mathbf{S}_{-1}^{\mathbb{C}}\left(\tau_{\mu}^{\lambda}\right)$ is simple if and only if $\lambda$ or $\mu$ is equal to $\varnothing$.

Proof. We first note that the composition factors of $\mathbf{S}_{-1}^{\mathbb{C}}\left(\tau_{\mu}^{\lambda}\right)$ are all of the form $\mathbf{D}_{-1}^{\mathbb{C}}\left(\tau_{\varnothing}^{\nu}\right)$ and so we need only consider 2-quotient separated partitions for the remainder of the proof. To see this, assume that $\lambda, \mu \in B_{k}(n)$ and that $\lambda$ is 2 -quotient separated, but $\mu$ is not. The partition $\mu$ must have at least $k+2$ complete ladders (as it is not 2-separated) and so $\ell(\mu) \geqslant \ell(\mathrm{R}(\mu)) \geqslant k+2$. On the other hand, $\lambda$ is 2-quotient separated and so it has at most $k+1$ complete ladders, $\ell(\mathrm{R}(\lambda)) \leqslant k+1$. Now, $\ell(\mu)>\ell(R(\lambda))$ implies that $\mu \not R(\lambda)$. The result follows from Theorem 1.4 .

As in Section 2, it suffices to restrict our attention to the dimensions of "weight spaces" given by the ladder tableaux for 2-regular partitions (in other words, we consider the dimensions of $\left.e\left(\tau_{\varnothing}^{\alpha}\right) \mathbf{S}_{-1}^{\mathbb{C}}\left(\tau_{\mu}^{\lambda}\right)\right)$. We further note that, if $\mu=\varnothing$, then $|\mu|=0$ and therefore the character of $e\left(\tau_{\varnothing}^{\alpha}\right) \mathbf{S}_{-1}^{\mathbb{C}}\left(\tau_{\varnothing}^{\nu}\right)$, given in equation (4.1), is bar-invariant for all $\alpha$ and $\nu$; this implies that $\mathbf{S}_{-1}^{\mathbb{C}}\left(\tau_{\varnothing}^{\nu}\right)=\mathbf{D}_{-1}^{\mathbb{C}}\left(\tau_{\varnothing}^{\nu}\right)$ by Theorem 2.10. In particular, we note that

$\operatorname{Dim}_{t}\left(e\left(\tau_{\varnothing}^{\alpha}\right) \mathbf{S}_{-1}^{\mathbb{C}}\left(\tau_{\varnothing}^{\alpha}\right)\right)=\operatorname{Dim}_{t}\left(e\left(\tau_{\varnothing}^{\alpha}\right) \mathbf{D}_{-1}^{\mathbb{C}}\left(\tau_{\varnothing}^{\alpha}\right)\right)=\left[\operatorname{Lad}\left(\tau_{\varnothing}^{\alpha}\right)\right]_{t} !=\left[\alpha^{T}\right]_{t} !^{2} \times[k]_{t} !$ as an obvious special case of equation (4.1), using Proposition 2.11(ii). For an arbitrary 2-separated simple module, this implies that

$$
\begin{aligned}
\operatorname{Dim}_{t}\left(e\left(\tau_{\varnothing}^{\alpha}\right) \mathbf{S}_{-1}^{\mathbb{C}}\left(\tau_{\varnothing}^{\nu}\right)\right) & =\operatorname{Dim}_{t}\left(e\left(\tau_{\varnothing}^{\alpha}\right) \mathbf{D}_{-1}^{\mathbb{C}}\left(\tau_{\varnothing}^{\nu}\right)\right) \\
& =\left|\operatorname{SStd}\left(\nu^{T}, \alpha^{T}\right)\right| \times \operatorname{Dim}_{t}\left(e\left(\tau_{\varnothing}^{\alpha}\right) \mathbf{D}_{-1}^{\mathbb{C}}\left(\tau_{\varnothing}^{\alpha}\right)\right) .
\end{aligned}
$$

Finally, we have that

$$
\left|\operatorname{SStd}\left(\lambda^{T} \cup \mu, \alpha^{T}\right)\right|=\sum_{\nu} c\left(\nu^{T}, \lambda^{T}, \mu\right)\left|\operatorname{SStd}\left(\nu^{T}, \alpha^{T}\right)\right|
$$

by the definition of the Littlewood-Richardson coefficients, hence equation (4.2) holds. Equation (4.3) follows immediately by induction on the dominance ordering (as in the LLT algorithm of Subsection 2.1). 
Proof of Theorem 4.1. We let $\tau:=\tau(k)$ and we assume for notational purposes that $k$ is even; the $k$ odd case is identical except that the residues 0 and 1 must be transposed. We let $w=|\alpha|=|\lambda|+|\mu|$. Given $\mathrm{s} \in \operatorname{Std}\left(\tau_{\mu}^{\lambda}\right)$, we have that $\operatorname{res}(\mathrm{s})=\operatorname{res}\left(\mathrm{L}_{\varnothing}^{\alpha}\right)$ if and only if $\mathrm{s}=\mathrm{t} \circ \mathrm{u}$ where

$$
\begin{aligned}
\operatorname{res}(\mathrm{t}) & =\left(0^{1}, 1^{2}, 0^{3}, 1^{4}, \ldots, 1^{k}\right) \quad \operatorname{res}(\mathrm{u}) \\
& =(\underbrace{0,0, \ldots, 0}_{\alpha_{1}^{T} \text { times }}, \underbrace{1,1, \ldots, 1}_{\alpha_{1}^{T} \text { times }}) \circ(\underbrace{0,0, \ldots, 0}_{\alpha_{2}^{T} \text { times }}, \underbrace{1,1, \ldots, 1}_{\alpha_{2}^{T} \text { times }}) \cdots,
\end{aligned}
$$

and we let $\operatorname{Std}_{\alpha}\left(\tau_{\mu}^{\lambda} \backslash \tau\right)$ denote the set of all tableaux $\mathrm{u} \in \operatorname{Std}\left(\tau_{\mu}^{\lambda} \backslash \tau\right)$ of the required residue sequence. We have that $\operatorname{deg}\left(\sum_{\mathrm{t} \in \mathbf{L}^{\tau}} t^{\operatorname{deg}(\mathrm{t})}\right)=[k]_{t} !$. All that remains is to show that

$$
\sum_{\mathbf{s} \in \operatorname{Std}_{\alpha}\left(\tau_{\mu}^{\lambda} \backslash \tau\right)} t^{\operatorname{deg}(\mathrm{s})}=\left|\operatorname{SStd}\left(\lambda^{T} \cup \mu, \alpha^{T}\right)\right| \times\left[\alpha^{T}\right]_{t}^{2} \times t^{|\mu|} .
$$

Given an integer $j \in\{1,2, \ldots, 2 w\}$ we have that there exists a unique corresponding integer $a(j) \in\{1, \ldots, w\}$ such that

$$
1+2 \sum_{i=1}^{a(j)-1}\left|\alpha_{i}^{T}\right| \leqslant j \leqslant 1+2 \sum_{i=1}^{a(j)}\left|\alpha_{i}^{T}\right|
$$

these integers will record the weight of the semistandard tableaux in the statement of equation (4.4). Namely, we record a skew-tableau s by placing both the usual entry $j \in\{1,2, \ldots, 2 w\}$ but we also add a subscript $a(j)$. An example is depicted on the left-hand side of Figure 7 . Recall that we can think of the partition $\tau_{\mu}^{\lambda}$ as being obtained by adding (2)-dominoes to the right of $\tau$ and $\left(1^{2}\right)$-dominoes to the bottom of $\tau$ in an intuitive fashion demonstrated in Figure 6. Take the partition $\tau$ and add a total of $\alpha_{1}^{T}$ nodes of residue 0 ; the resulting partition has precisely $\alpha_{1}^{T}$ addable 1 -nodes $X_{1}, \ldots, X_{\alpha_{1}}$ : namely, those which belong to the (2)- and ( $\left.1^{2}\right)$-dominoes containing the nodes $X_{1}, \ldots, X_{\alpha_{1}}$. Repeating this observation as necessary, we deduce that any two nodes in the same domino of a tableau $\mathrm{s} \in \operatorname{Std}_{\alpha}\left(\tau_{\mu}^{\lambda} \backslash \tau\right)$ have the same subscript. Furthermore, we note that the fact that the residue sequence is of the form

$$
(\underbrace{0,0, \ldots, 0}_{\alpha_{1}^{T} \text { times }}, \underbrace{1,1, \ldots, 1}_{\alpha_{1}^{T} \text { times }}) \circ(\underbrace{0,0, \ldots, 0}_{\alpha_{2}^{T} \text { times }}, \underbrace{1,1, \ldots, 1}_{\alpha_{2}^{T} \text { times }}) \circ \cdots
$$

implies that no two (2)-dominoes of the same subscript can be added in the same row and no two $\left(1^{2}\right)$-dominoes of the same subscript can be added in the same column. Therefore we obtain a well-defined map

$$
\varphi: \operatorname{Std}_{\alpha}\left(\tau_{\mu}^{\lambda} \backslash \tau\right) \longmapsto \operatorname{SStd}\left(\lambda^{T} \cup \mu, \alpha^{T}\right)
$$

given by scaling the sizes of all the dominoes by $1 / 2$, conjugating $\lambda$, and recording only the subscripts (i.e., deleting the integers $\{1, \ldots, 2 w\}$ ). An example is depicted in Figure 7.

All that remains to show is that

$$
\sum_{\{\mathbf{s} \mid \varphi(\mathbf{s})=\mathrm{S}\}} t^{\operatorname{deg}(\mathbf{s})}=\left[\alpha^{T}\right]_{t} !^{2} \times t^{|\mu|}
$$



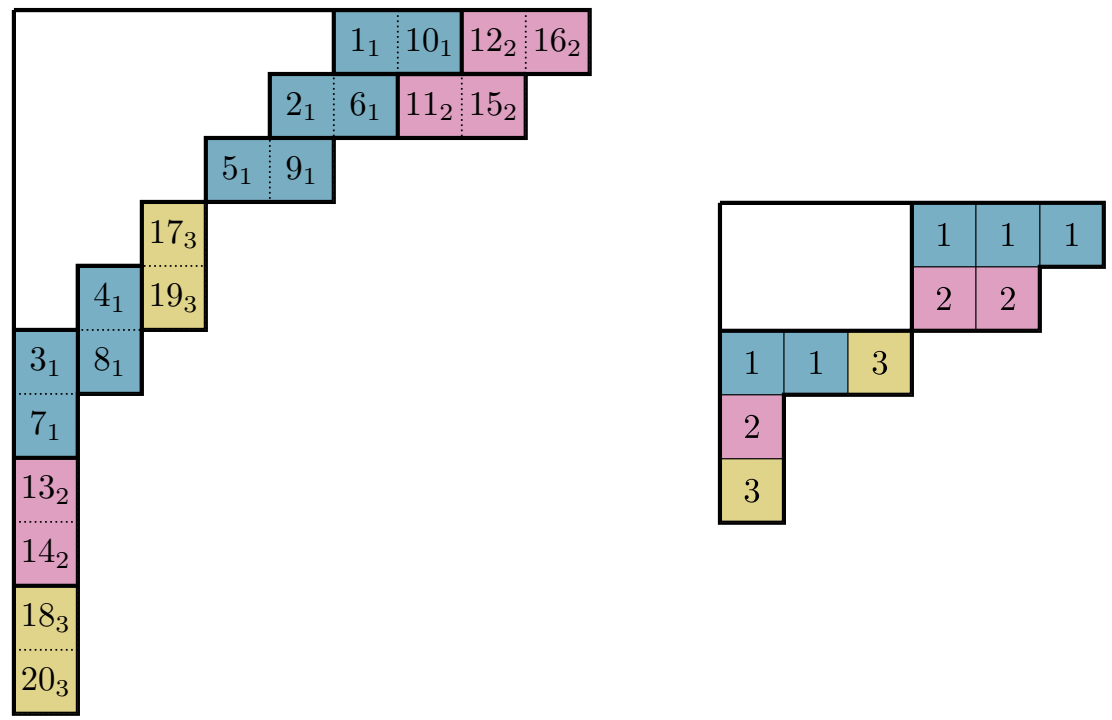

FiguRE 7. A $\left(3^{2}, 2,1^{2}\right)$-decorated standard tableau of shape $\tau_{(3,1,1)}^{(2,2,1)} \backslash \tau$ and the corresponding element of $\operatorname{SStd}((3,2) \cup$ $(3,1,1),(5,3,2))$. The associated sequence of partitions (as in equation (4.6) ) is $\tau \subset \tau_{(2)}^{\left(1^{3}\right)} \subset \tau_{(2,1)}^{\left(2^{2}, 1\right)} \subset \tau_{\left(3,1^{2}\right)}^{\left(2^{2}, 1\right)}$.

for any $\mathrm{S} \in \operatorname{SStd}\left(\lambda^{T} \cup \mu, \alpha^{T}\right)$. The set $\{\mathrm{s} \mid \varphi(\mathrm{s})=\mathrm{S}\}$ consists of an orbit

$$
\mathfrak{S}_{\alpha_{1}} \times \mathfrak{S}_{\alpha_{1}} \times \mathfrak{S}_{\alpha_{2}} \times \mathfrak{S}_{\alpha_{2}} \times \ldots
$$

of standard tableaux. In other words, $\mathrm{s}, \mathrm{t}$ are $\operatorname{such} \varphi(\mathrm{s})=\varphi(\mathrm{t})$ if and only if they differ by permuting nodes whose subscripts and residues are both matching. Therefore, we have that

$$
\sum_{\{\mathbf{s} \mid \varphi(\mathbf{s})=\mathrm{S}\}} 1^{\operatorname{deg}(\mathbf{s})}=\left(\alpha_{1}^{T} ! \alpha_{2}^{T} ! \ldots\right)^{2}=\left.\left(\left[\alpha^{T}\right]_{t} !^{2}\right)\right|_{t=1},
$$

and so the ungraded version of equation (4.5) follows.

It remains to consider the grading. We first cut the diagram of any 2-separated partition $\tau_{\mu}^{\lambda}$ into four regions by drawing a vertical line immediately after the $\mu_{1}$ th column of $\tau_{\mu}^{\lambda}$ and a horizontal line immediately below the $\lambda_{1}^{T}$ th row. An example is depicted in Figure 8. We label the three of the four quarters of the diagram $X:=X\left(\tau_{\mu}^{\lambda}\right), Y:=Y\left(\tau_{\mu}^{\lambda}\right)$, and $Z:=Z\left(\tau_{\mu}^{\lambda}\right)$ as suggested in Figure 8 , The intersection of $\tau_{\mu}^{\lambda}$ with the region $Y$ is equal to the staircase partition of width $\rho_{1}-\mu_{1}-\lambda_{1}^{T}$; we set $\rho_{Y}:=\left[\tau_{\mu}^{\lambda}\right] \cap Y$.

We shall calculate the left-hand-side of equation (4.5) by peeling off a row of $\alpha$ at a time, in a manner which we now make precise. Fix $\alpha \in \mathscr{P}_{n}$ a partition and set $\ell=\ell\left(\alpha^{T}\right)$ and $\mathrm{S} \in \operatorname{SStd}\left(\lambda^{T} \cup \mu, \alpha^{T}\right)$. We define

$$
\left.\mu_{j}^{(i)}=\mid\{(j, c) \in \mu \mid \mathrm{S}(j, c) \leqslant i\}\right\}\left|\quad \lambda_{j}^{(i)}=\right|\{(r, j) \in \lambda \mid \mathrm{S}(r, j) \leqslant i\} \mid
$$




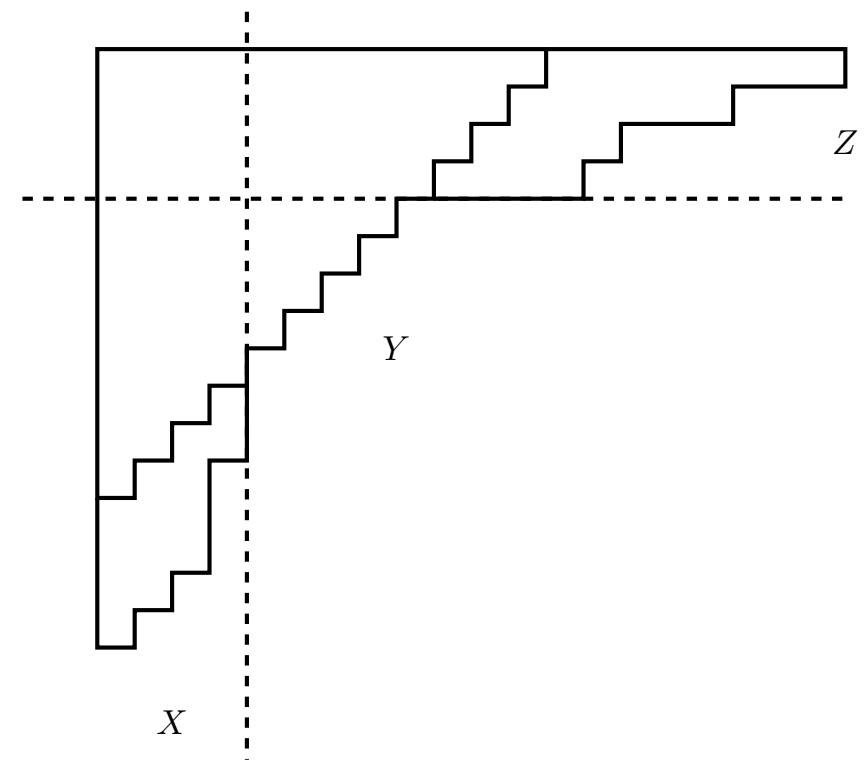

FIgURE 8. Dividing the partition $\tau_{\mu}^{\lambda}$ into regions $X:=X\left(\tau_{\mu}^{\lambda}\right)$, $Y:=Y\left(\tau_{\mu}^{\lambda}\right)$ and $Z:=Z\left(\tau_{\mu}^{\lambda}\right)$. In this case $\rho_{Y}$ is the copy of the partition $(4,3,2,1)$ in region $Y$.

for $1 \leqslant i \leqslant \ell(\alpha)$. We set $\mu(i)=\left(\mu_{1}^{(i)}, \ldots, \mu_{\ell}^{(i)}\right)$ and $\lambda(i)=\left(\lambda_{1}^{(i)}, \ldots, \lambda_{\ell}^{(i)}\right)$. We consider the associated sequence of partitions

$$
\tau=\tau_{\mu(0)}^{\lambda(0)} \subseteq \tau_{\mu(1)}^{\lambda(1)} \subseteq \tau_{\mu(2)}^{\lambda(2)} \cdots \subseteq \tau_{\mu(\ell)}^{\lambda(\ell)}=\tau_{\mu}^{\lambda},
$$

an example is given in Figure 7 Setting $\alpha^{T}=\left(a_{1}, \ldots, a_{\ell}\right)$, we will show that

$$
\sum_{\operatorname{s\in Std}_{\left(a_{m}\right)}\left(\tau_{\mu(m)}^{\lambda(m)} \backslash \tau_{\mu(m-1)}^{\lambda(m-1)}\right)} t^{\operatorname{deg}(\mathrm{s})}=\left[a_{m}\right]_{t} !^{2} \times t^{|\mu(m)|-|\mu(m-1)|},
$$

and hence deduce the result. Clearly we can calculate the degree contribution of the node $(r, c)$ to the tableau

$$
\mathrm{s} \in \operatorname{Std}_{\left(a_{m}\right)}\left(\tau_{\mu(m)}^{\lambda(m)} \backslash \tau_{\mu(m-1)}^{\lambda(m-1)}\right)
$$

by considering the addable/removable nodes above $(r, c)$ from the regions

$$
X_{m}:=X\left(\tau_{\mu(m)}^{\lambda(m)}\right) \quad Y_{m}:=Y\left(\tau_{\mu(m)}^{\lambda(m)}\right) \quad Z_{m}:=Z\left(\tau_{\mu(m)}^{\lambda(m)}\right)
$$

separately. We let $\operatorname{deg}_{X_{m}}(r, c), \operatorname{deg}_{Y_{m}}(r, c)$ and $\operatorname{deg}_{Z_{m}}(r, c)$ denote these respective contributions. We first consider the contribution from $Y_{m}$. By definition, the sum total

$$
\operatorname{deg}_{Y_{m}}(\mathrm{~s})=\sum_{1 \leqslant k \leqslant 2 m} \operatorname{deg}_{Y_{m}} \mathrm{~s}^{-1}(k)
$$

is independent of the tableau $\mathrm{s} \in \operatorname{Std}\left(\tau_{\mu(m)}^{\lambda(m)} \backslash \tau_{\mu(m-1)}^{\lambda(m-1)}\right)$. For $\rho_{Y_{m}}$ a staircase partition of $p(p+1) / 2$, we have that

$$
\left|\operatorname{Rem}_{0}\left(\rho_{Y_{m}}\right)\right|=0 \quad\left|\operatorname{Rem}_{1}\left(\rho_{Y_{m}}\right)\right|=p \quad\left|\operatorname{Add}_{0}\left(\rho_{Y_{m}}\right)\right|=p+1 \quad\left|\operatorname{Add}_{1}\left(\rho_{Y_{m}}\right)\right|=0 .
$$


Therefore $\operatorname{deg}_{Y_{m}}(\mathrm{~s})$ is equal to the number of $\left(1^{2}\right)$-bricks in region $X_{m} \cap \tau_{\mu(m)}^{\lambda(m)} \backslash$ $\tau_{\mu(m-1)}^{\lambda(m-1)}$. In other words $\operatorname{deg}_{Y_{m}}(\mathrm{~s})=|\mu(m)|-|\mu(m-1)|$.

Finally, it remains to prove that

$$
\sum_{\mathbf{s} \in \operatorname{Std}_{\left(a_{m}\right)}} t^{\left.\operatorname{deg}_{\mu(m)}^{\lambda(m)} \backslash \tau_{\mu(m-1)}^{\lambda(m-1)}\right)}{ }^{\operatorname{Xoz}_{m}(\mathrm{~s})}=\left[a_{m}\right]_{t} !^{2} .
$$

We set $\operatorname{Std}_{m}:=\operatorname{Std}\left(\rho\left(a_{m}\right) \backslash \rho\left(a_{m}-1\right)\right)$. It is easy to see that

$$
\sum_{\mathbf{s} \in \operatorname{Std}_{\left(a_{m}\right)}} t^{\left.\operatorname{deg}_{\tau_{m} \cup(m)}^{\lambda(m)} \backslash \tau_{\mu(m-1)}^{\lambda(m-1)}\right)}\left(\sum_{\mathbf{s} \in \operatorname{Std}_{m}} t^{\operatorname{deg}(\mathbf{s})}\right) \times\left(\sum_{\mathbf{s} \in \operatorname{Std}_{m}} t^{\operatorname{deg}(\mathbf{s})}\right),
$$

where the first/second multiplicand on the right-hand-side counts the contribution of all the residue 0 -boxes/1-boxes respectively. We set $\square_{j}=\left(j, a_{m}+1-j\right) \in \rho\left(a_{m}\right)$ for $1 \leqslant j \leqslant a_{m}$. We have that

$$
\begin{aligned}
\sum_{\substack{1 \leqslant j \leqslant a_{m} \\
\left\{\mathbf{s} \in \operatorname{Std}_{m} \mid \mathbf{s}^{-1}\left(a_{m}\right)=\square_{j}\right\}}} t^{\operatorname{deg}(\mathrm{s})} & =\sum_{\substack{\mathrm{t} \in \operatorname{Std}_{m-1} \\
1 \leqslant j \leqslant a_{m}}} t^{a_{m}-j} \times t^{1-j} \times t^{\mathrm{deg}(\mathrm{t})} \\
& =\sum_{1 \leqslant j \leqslant a_{m}} t^{a_{m}+1-2 j}\left[a_{m}-1\right]_{t} !
\end{aligned}
$$

which is equal to $\left[a_{m}\right]_{t}$ !. Here the first equality follows by considering both the degree of the node $\mathrm{s}^{-1}\left(a_{m}\right)$ (which is equal to $1-j$ ) and the resulting shift to the degrees of each of the $\left(a_{m}-j\right)$ nodes below s ${ }^{-1}\left(a_{m}\right)$. The second equality holds by induction. Therefore the result follows.

\section{Kronecker COEFFicients And SAXl's CONJECture}

Let $\lambda, \mu, \nu$ be partitions of $n$. For the remainder of the paper, we will let $\mathbf{D}^{\mathbb{k}}(\lambda)$ denote the unquantised simple $\mathbb{k} \mathfrak{S}_{n}$-module. We define the Kronecker coefficients $g(\lambda, \mu, \nu)$ to be the coefficients in the expansion

$$
\mathbf{D}^{\mathbb{C}}(\lambda) \otimes \mathbf{D}^{\mathbb{C}}(\mu)=\bigoplus_{\nu \vdash n} g(\lambda, \mu, \nu) \mathbf{D}^{\mathbb{C}}(\nu) .
$$

We now recall Saxl's conjecture concerning the positivity of these coefficients. We let $\chi^{\lambda}$ denote the complex irreducible $\mathfrak{S}_{n}$-character to the partition $\lambda$ of $n$, i.e., the character of the Specht module $\mathbf{S}^{\mathbb{C}}(\lambda)$.

Saxl's conjecture. Let $n=k(k+1) / 2$ and $\rho=(k, k-1, \ldots, 2,1)$. For all $\lambda \vdash n$, the multiplicity of $\chi^{\lambda}$ in the Kronecker product $\chi^{\rho} \cdot \chi^{\rho}$ is strictly positive.

In HSTZ13, Heide, Saxl, Tiep and Zalesski verified that for almost all finite simple groups of Lie type the square of the Steinberg character contains all irreducible characters as constituents. They also conjectured that for all alternating groups there is some irreducible character with this property. For symmetric groups $\mathfrak{S}_{n}$ to triangular numbers $n$, Saxl then suggested the candidate $\chi^{\rho}$ as stated above. Saxl's conjecture has been attacked by algebraists and complexity theorists using a variety of combinatorial and probabilistic methods [Bes18, Ike15, LS17, PPV16]. From our perspective, a particularly useful result is the following.

Theorem 5.1 (Ike15, Theorem 2.1]). Let $n=k(k+1) / 2$ and $\rho=(k, k-1, \ldots, 2,1)$. If $\lambda$ is a partition of $n$ such that $\lambda \triangleq \rho$ or $\lambda \triangleq \rho$, then $g(\rho, \rho, \lambda)>0$. 
We let $\mathbb{k}$ be a field of characteristic 2 . We keep the notation $\rho=\rho(k)$ and $n=k(k+1) / 2$, and we note that $\mathbf{D}^{\mathbb{k}}(\rho)=\mathbf{S}^{\mathbb{k}}(\rho)=\mathbf{P}^{\mathbb{k}}(\rho)$ is a simple projective

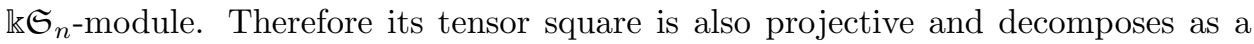
direct sum of indecomposable projective modules labelled by 2-regular partitions; we let $G(\rho, \rho, \nu)$ denote the corresponding coefficients as follows

$$
\mathbf{D}^{\mathbb{k}}(\rho) \otimes \mathbf{D}^{\mathbb{k}}(\rho)=\bigoplus_{\nu \vdash_{2} n} G(\rho, \rho, \nu) \mathbf{P}^{\mathbb{k}}(\nu) .
$$

Equivalently, on the level of complex characters we have for the irreducible character $\chi^{\rho}$ the decomposition of its square into characters $\xi^{\nu}$ to projective indecomposable modules (i.e., to integral lifts of the projective modules at characteristic 2 ):

$$
\left(\chi^{\rho}\right)^{2}=\bigoplus_{\nu \vdash_{2} n} G(\rho, \rho, \nu) \xi^{\nu} .
$$

We wish to pass information back and forth between the 2-modular coefficients $G(\lambda, \mu, \nu)$ and the Kronecker coefficients $g(\lambda, \mu, \nu)$ in order to make headway on Saxl's conjecture. The following observation is immediate

$$
g(\rho, \rho, \lambda)=\sum_{\nu} G(\rho, \rho, \nu) d_{\lambda, \nu}
$$

We first want to explain how to apply this to obtain positivity for new classes of Kronecker coefficients. Recall from Subsection 1.3 that the 2-blocks of $\mathfrak{S}_{n}$ are parameterized by the common 2-core of the partitions labelling the simple modules in characteristic 2 and the Specht modules in the block, together with the weight. Further recall from Subsection 1.1 that the decomposition matrix for each block is unitriangular with respect to the dominance ordering. In particular, the most dominant partition, $\tau_{\varnothing}^{(w)}$, in a given 2-block of weight $w$ labels a Specht module with simple reduction mod 2. Ikenmeyer's result implies that $S^{\mathbb{C}}\left(\tau_{\varnothing}^{(w)}\right)$ appears with positive multiplicity; therefore $S^{\mathbb{k}}\left(\tau_{\varnothing}^{(w)}\right)$ appears as a subquotient of some projective $\mathbb{k} \mathfrak{S}_{n}$-module; by maximality we know that the only projective containing $S^{\mathbb{k}}\left(\tau_{\varnothing}^{(w)}\right)$ as a subquotient is, in fact, $P^{\mathbb{k}}\left(\tau_{\varnothing}^{(w)}\right)$ itself. Thus $P^{\mathbb{k}}\left(\tau_{\varnothing}^{(w)}\right)$ appears as a direct summand in equation (5.1) and so $G\left(\rho, \rho, \tau_{\varnothing}^{(w)}\right)>0$.

Hence, any non-zero entry of the first column (labelled by $\tau_{\varnothing}^{(w)}$ ) of the 2-decomposition matrix of any 2-block corresponds to a non-zero Kronecker coefficient. This allows us to verify Kronecker positivity in Saxl's conjecture for two new infinite families of partitions:

Theorem 5.2. Let $n=k(k+1) / 2, \rho=\rho(k)$ and $\lambda \vdash n$ such that $\chi^{\lambda}$ is of height 0 . Then $g(\rho, \rho, \lambda)>0$. In particular, all $\chi^{\lambda}$ of odd degree are constituents of the Saxl square.

Proof. Let $B$ be the 2-block of $\mathfrak{S}_{n}$ to which $\chi^{\lambda}$ belongs. Because $\chi^{\lambda}$ is a character of height 0 , the modulo 2 reduction $\mathbf{S}^{\mathbb{k}}(\lambda)$ must have a composition factor of height zero and this composition factor must appear with odd multiplicity (simply by comparing the dimensions, see Subsection 1.4). By Theorem 1.13, the 2-block $B$ contains a unique simple module $D=\mathbf{D}^{\mathbb{k}}(\mu)$ of height 0 , with $\mu=\tau_{\varnothing}^{(w)}=\tau+(2 w)$ the most dominant partition belonging to the block. The discussion preceding the theorem now implies

$$
g(\rho, \rho, \lambda) \geq d_{\lambda, \tau+(2 w)}>0 .
$$


Since we get the large number $k_{0}(B)$ of height 0 irreducible characters for each 2-block $B$, this constitutes quite a large class of constituents in the Saxl square.

Example 5.3. We have already seen (in Example 1.12) that the block $B_{3}(36)$ contains 1024 height 0 characters, which all appear in Saxl's tensor square.

Example 5.4. We consider the partition $\lambda=(9,8,3,2,2,2,1,1) \vdash 28$. This is the first of the four partitions pictured in Figure 2. In this case, the desired positivity of $g(\rho(7), \rho(7), \lambda)$ cannot be deduced using the available non-vanishing criteria in the literature Bes18, PPV16], and $\lambda$ is incomparable to $\rho$ in the dominance order, so [ke15] does not apply. The character $\chi^{\lambda}$ belongs to the 2-block of weight $w=11$ and 2-core $\rho(3)$, and it is of height 0 . Instead of computing the degree explicitly, this can also be seen by applying one of the combinatorial descriptions for labels of height 0 characters, e.g., the one due to GMT18 recalled in Subsection 1.4. Finally, referring forward in this paper: we remark that $(9,8,3,2,2,2,1,1)$ is not 2 -separated. Thus Theorem 5.2 provides us with constituents of the Saxl square that cannot be deduced using any other results in the literature.

For the second new family, we will also need to apply our new results on Specht modules for the Hecke algebra.

Theorem 5.5. For $\tau_{\left(1^{\ell}\right)}^{(m)}$ any framed staircase partition of $n=k(k+1) / 2$, we have that $g\left(\rho, \rho, \tau_{\left(1^{\ell}\right)}^{(m)}\right)>0$.

Proof. Let $w=\ell+m$ be the weight of the 2-block $B$ to which $\tau_{\left(1^{\ell}\right)}^{(m)}$ belongs. We have that $\tau_{\varnothing}^{(w)}$ is the most dominant partition in $B$ and so the corresponding Specht module is simple. Now

$$
\left[\mathbf{S}_{-1}^{\mathbb{k}}\left(\tau_{\left(1^{\ell}\right)}^{(m)}\right): \mathbf{D}_{-1}^{\mathbb{k}}\left(\tau_{\varnothing}^{(w)}\right)\right] \geqslant\left[\mathbf{S}_{-1}^{\mathbb{C}}\left(\tau_{\left(1^{\ell}\right)}^{(m)}\right): \mathbf{D}_{-1}^{\mathbb{C}}\left(\tau_{\varnothing}^{(w)}\right)\right]=c\left(\left(1^{w}\right),\left(1^{m}\right),\left(1^{\ell}\right)\right)=1>0
$$

and so the result follows by the discussion above.

For reasons that will soon become apparent, we now recall Carter's criterion explicitly.

Theorem 5.6 ([JM99]). We let $\mathbb{k}$ be a field of characteristic 2. Let $\lambda=\left(\lambda_{1}, \lambda_{2}, \ldots, \lambda_{\ell}\right)$ be a partition. Then the Specht module $\mathbf{S}^{\mathbb{k}}(\lambda)$ is simple if and only if one of the following conditions holds:

(i) $\lambda_{i}-\lambda_{i+1} \equiv-1$ modulo $2^{\ell_{2}\left(\lambda_{i+1}-\lambda_{i+2}\right)}$ for all $i \geqslant 1$;

(ii) the transpose partition, $\lambda^{T}$, satisfies $(i)$;

(iii) $\lambda=(2,2)$,

where here $\ell_{2}(k)$ is the least non-negative integer such that $k<2^{\ell_{2}(k)}$. We say that any partition as in ( $i$ ) satisfies Carter's criterion.

Example 5.7. The most dominant partition, $\tau_{\varnothing}^{(w)}$, in a 2-block of weight $w$ satisfies Carter's criterion.

Example 5.8. In a 2-block of weight $w=m(m+1) / 2$, we find the partition $\tau_{\varnothing}^{\rho(m)}$ that satisfies Carter's criterion.

If $\lambda$ is a 2-regular partition, then all the rows of $\lambda$ are of distinct length. It immediately follows that $\lambda \geqslant \rho$ and therefore $g(\rho, \rho, \lambda)>0$. If furthermore the partition $\lambda$ satisfies Carter's criterion, then by equation (1.2) we have that $\mathbf{P}^{\mathbb{k}}(\lambda)$ is 
the unique projective module in which $\mathbf{S}^{\mathbb{k}}(\lambda)$ appears as a composition factor of a Specht filtration. Putting these two statements together (in light of equation (5.2)) we obtain the following.

Proposition 5.9. Let $n=k(k+1) / 2$ and $\alpha \vdash n$. If $\alpha$ satisfies Carter's criterion, then we have that $G(\rho(k), \rho(k), \alpha)>0$.

The following result is immediate by equation (5.2). It is the key to all of our results on Kronecker positivity (as it relates this problem to that of determining the positivity of modular decomposition numbers) and vastly generalises Theorem 5.5 .

Theorem 5.10. Let $n=k(k+1) / 2$ and $\alpha \vdash n$. If there exists some $\beta$ satisfying Carter's criterion such that $d_{\alpha, \beta}=m>0$, then $g(\rho(k), \rho(k), \alpha) \geqslant m$. We refer to such a pair $(\alpha, \beta)$ as an $m$-Carter-Saxl pair.

We are now ready to use the results of Sections 3 and 4 toward the Kronecker problem.

Theorem 5.11. Let $w=k(k+1) / 2, n=w(2 w+1)$ and $\tau=\rho(2 w-1)$. Then for $\lambda, \mu$ any pair such that $c\left(\rho(k), \lambda^{T}, \mu\right)>0$ we have that

$$
g\left(\rho(2 w), \rho(2 w), \tau_{\mu}^{\lambda}\right) \geqslant c\left(\rho(k), \lambda^{T}, \mu\right)>0 .
$$

Proof. Clearly, $\rho(2 w)$ is a partition of $n=w(2 w+1)$. We restrict our attention to the block $B$ of weight $w$ in $\mathbb{k}_{n}$ with 2-core $\tau=\rho(2 w-1)$. For $\nu=\rho(k)$, the partition $\tau_{\varnothing}^{\nu}$ belongs to this block, and it satisfies Carter's criterion. Note that $c\left(\rho(k), \lambda^{T}, \mu\right)>0$ implies that $|\lambda|+|\mu|=w$, and $\tau_{\mu}^{\lambda}$ also belongs to $B$. Finally, we have that

$$
\left[\mathbf{S}_{-1}^{\mathbb{k}}\left(\tau_{\mu}^{\lambda}\right): \mathbf{D}_{-1}^{\mathbb{k}}\left(\tau_{\varnothing}^{\nu}\right)\right] \geqslant\left[\mathbf{S}_{-1}^{\mathbb{C}}\left(\tau_{\mu}^{\lambda}\right): \mathbf{D}_{-1}^{\mathbb{C}}\left(\tau_{\varnothing}^{\nu}\right)\right]=c\left(\rho(k), \lambda^{T}, \mu\right)>0,
$$

and the result follows from Theorem 5.10 .

We remark that none of the partitions above are covered by existing results in the literature. It is clear that they are not hooks or double-hooks and providing that neither $\lambda$ or $\mu$ is the empty partition, then these partitions are not comparable with $\rho(2 w)$ in the dominance order (as $\tau_{\mu}^{\lambda}$ is both wider and longer that $\rho(2 w)$ ).

An explicit new infinite family with unbounded Kronecker coefficients is given in the following corollary.

Corollary 5.12. For $w=k(k+1) / 2, n=w(2 w+1)$ and $\tau=\rho(2 w-1)$, we have that

$$
g\left(\rho(2 w), \rho(2 w), \tau_{(k-1,1)}^{\rho(k-1)}\right) \geqslant k-1
$$

Proof. We have

$$
c(\rho(k), \rho(k-1),(k-1,1))=k-1,
$$

from which the result follows from Theorem 5.11

Example 5.13. In particular, for $w=k(k+1) / 2, g\left(\rho(2 w), \rho(2 w), \tau_{\mu}^{\lambda}\right)>0$ for $\lambda, \mu$ any pair such that $\lambda+\mu^{T}=\rho(k)$. Examples of such partitions $\tau_{\mu}^{\lambda}$ are pictured in Figure 4

Example 5.14. Let $n=210$ and consider the 2-block of weight 20 with 2-core $\rho(19)$. There exist 35 Carter-Saxl pairs belonging to pairs $(\lambda, \mu)$ such that $\lambda+\mu^{T}$ is equal to either $(10)$ or $(4,3,2,1)$. There are many more Carter-Saxl pairs in this block. 
Finally, we conclude this section by remarking that we have only used positivity of decomposition numbers for the Hecke algebra over $\mathbb{C}$. These are the easiest decomposition numbers to calculate, but only provide lower bounds for the decomposition numbers of symmetric groups.

\section{More Semisimple Decomposable Specht modules}

Semisimplicity and decomposability of Specht modules has long been a subject of major interest: the highlight being the recent progress on the classification of simple Specht modules for symmetric groups and their Hecke algebras [FL13, Jam78, JLM06, JM99, Fay05, JM96, Lyl07, JM97, Fay04, FL09, Fay10, Progress towards understanding the wider family of semi-simple and decomposable modules has been snail-like in comparison [Mur80, Spe14,CMT04, DF12, Rou08b, FS16] and reserved solely to near-hook partitions. All examples of decomposable Specht modules discovered to date have been labelled by 2 -separated partitions. Our Theorem A proves that for any 2-separated partition, the corresponding Specht module for the algebra $H_{-1}^{\mathbb{C}}(n)$ is decomposable. It is natural to ask whether the converse is true: are all decomposable Specht modules for $H_{-1}^{\mathbb{C}}(n)$ and more generally $H_{-1}^{\mathbb{k}}(n)$ indexed by 2-separated partitions?. In [DF12, Section 8.2], Dodge and Fayers asked exactly this question for the symmetric group with char $\mathbb{k}=2$. In this section, we provide counterexamples to this question for the Hecke algebra.

6.1. Two new infinite families of decomposable Specht modules. Given $k \in \mathbb{N}$ and $l \in 2 \mathbb{N}+1$, we define $\alpha_{k} \vdash(k+2)^{2}-4$ and $\beta_{l} \vdash(l+2)^{2}-2$, respectively, to be the partitions

$$
\alpha_{k}=\left((k+2)^{k}, k^{2}\right), \quad \beta_{l}=\left(l+3,(l+2)^{l-1}, l^{2}, 1\right) .
$$

For example, the Young diagrams of the partitions $\alpha_{5}$ and $\beta_{5}$, along with their residues, are drawn as follows:

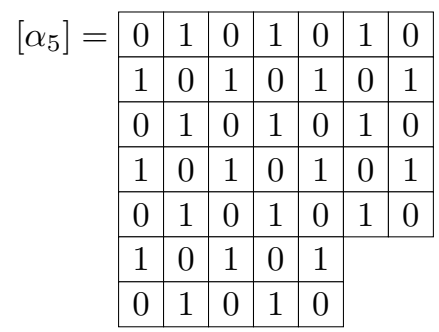

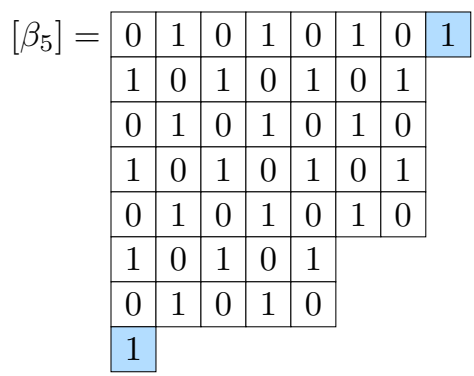

In the arXiv appendix to this paper, we prove that

$$
\mathbf{S}_{-1}^{\mathbb{C}}\left(\alpha_{[k]}\right) \text { and } \mathbf{S}_{-1}^{\mathbb{C}}\left(\beta_{[l]}\right)
$$

are decomposable for all $k \geqslant 1$ and odd $l \geq 1$. The graded composition factors of these modules for $1 \leqslant k, l \leqslant 5$ can be computed with the Hecke package in GAP (and shown to be concentrated in one degree). Thus decomposability and semisimplicity can be deduced from Theorem 3.2. These provide the first examples of decomposable Specht modules indexed by partitions which are not 2 -separated. Thus, while our Theorem A provides the largest family of decomposable (and semisimple) Specht modules discovered to date, it is worth noting that our list is not exhaustive. 
In the arXiv Appendix, we show that both Specht modules have a direct summand equal to a (different) simple Specht module. Namely,

$$
\begin{aligned}
& \mathbf{S}_{-1}^{\mathbb{C}}\left((k+4)^{k}\right) \cong \mathbf{D}_{-1}^{\mathbb{C}}(2 k+3,2 k+1,2 k-1, \ldots, 9,7,5) \text { is a direct summand of } \\
& \mathbf{S}_{-1}^{\mathbb{C}}\left(\alpha_{[k]}\right) .
\end{aligned}
$$

The proof of decomposability is not difficult, but it does involve twenty pages of extensive calculations. The basic idea is to (1) show that

$$
\operatorname{Hom}_{H_{-1}^{\mathbb{C}}(n)}\left(\mathbf{S}_{-1}^{\mathbb{C}}\left((k+4)^{k}\right), \mathbf{S}_{-1}^{\mathbb{C}}\left(\alpha_{[k]}\right)\right) \neq 0
$$

using results on semistandard homomorphisms and (2) prove that

$$
\left[\mathbf{S}_{-1}^{\mathbb{C}}\left(\alpha_{[k]}\right): \mathbf{D}_{-1}^{\mathbb{C}}(2 k+3,2 k+1,2 k-1, \ldots, 9,7,5)\right] \leqslant 1
$$

by counting corresponding coloured tableaux. One hence deduces that this simple composition factor occurs exactly once as a composition factor but in both the head and the socle of $\mathbf{S}_{-1}^{\mathbb{C}}\left(\alpha_{[k]}\right)$, and thus is a direct summand. We refer the reader to the arXiv appendix for more details.

Conjecture 6.1. For $k \in \mathbb{N}$, we set $\mathrm{C}\left(\alpha_{k}\right)=(2 k+3,2 k+1,2 k-1, \ldots, 9,7,5)$. Then we expect that

$$
\mathbf{S}_{-1}^{\mathbb{C}}\left(\alpha_{k}\right)=\mathbf{D}_{-1}^{\mathbb{C}}\left(\mathrm{R}\left(\alpha_{k}\right)\right)\left\langle w\left(\alpha_{k}\right) / 2\right\rangle \oplus \mathbf{D}_{-1}^{\mathbb{C}}\left(\mathrm{C}\left(\alpha_{k}\right)\right)\left\langle w\left(\alpha_{k}\right) / 2\right\rangle .
$$

By 1-induction, we conjecture the direct sum decomposition of $\mathbf{S}_{-1}^{\mathbb{C}}\left(\beta_{[l]}\right)$.

Conjecture 6.2. For $l \in 2 \mathbb{N}+1$, we set $\mathrm{C}\left(\beta_{l}\right)=(2 l+3,2 l+1,2 l-1, \ldots, 9,7,6,1)$. Then we expect that

$$
\mathbf{S}_{-1}^{\mathbb{C}}\left(\beta_{l}\right)=\mathbf{D}_{-1}^{\mathbb{C}}\left(\mathrm{R}\left(\beta_{l}\right)\right)\left\langle w\left(\beta_{l}\right) / 2\right\rangle \oplus \mathbf{D}_{-1}^{\mathbb{C}}\left(\mathrm{C}\left(\beta_{l}\right)\right)\left\langle w\left(\beta_{l}\right) / 2\right\rangle .
$$

6.2. Other decomposable Specht modules. We are indebted to Matt Fayers for sharing the following examples (which he discovered by computer) after we posited that the two families in Subsection 6.1 might be the only counterexamples to the quantised version of his question [DF12, Section 8.2].
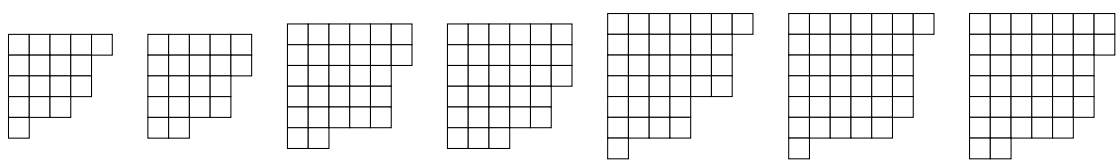

FiguRE 9. More partitions labelling semisimple Specht modules. The graded composition factors of these modules can be computed with the Hecke package in GAP (and shown to be concentrated in one degree). Thus decomposability and semisimplicity are deduced from Theorem 3.2 .

We hope that the examples in Figure 9 serve as inspiration for further work towards a classification of semisimple Specht modules. Several more examples can be obtained from those in Figure 9 by $i$-induction for $i=0,1$ (analogously to Subsection 6.1) namely: $\left(8,7,6^{2}, 4^{2}, 2,1\right),\left(7^{3}, 6^{3}, 3\right)$ and $\left(8,7^{3}, 6^{2}, 4,1\right)$. Finally we have one partition which breaks the mould: $\left(6,5^{3}, 3,1\right)$ which is the only partition in this section not equal to its own conjugate. 
6.3. Patterns. The examples of Subsections 6.1 and 6.2 do share several striking similarities. Firstly, all the examples in Subsections 6.1 and 6.2 have a direct summand which is isomorphic to a simple Specht module. Secondly, all those of Subsection 6.2 decompose as a direct sum of simples concentrated in one degree and so are semisimple by Theorem 3.2. We conjecture this is also true of the infinite families in Theorem 3.2. It is interesting to speculate whether the converse of Theorem 3.2 is also true: is semisimplicity of a Specht module equivalent to its composition factors being focused in one degree?

\section{ACKNOWLEDGMENTS}

We would like to thank Matt Fayers for helpful discussions on simple Specht modules and for sharing his extensive computer calculations which were fundamental in writing this paper. We also wish to thank Liron Speyer for playing "match-maker" in this collaboration. We are also very grateful to the referees for their helpful comments and suggestions.

\section{REFERENCES}

[BB17] Christine Bessenrodt and Christopher Bowman, Multiplicity-free Kronecker products of characters of the symmetric groups, Adv. Math. 322 (2017), 473-529, DOI 10.1016/j.aim.2017.10.009. MR.3720803

[Bes18] Christine Bessenrodt, Critical classes, Kronecker products of spin characters, and the Saxl conjecture, Algebr. Comb. 1 (2018), no. 3, 353-369, DOI 10.5802/alco. MR3856528

[BGS96] Alexander Beilinson, Victor Ginzburg, and Wolfgang Soergel, Koszul duality patterns in representation theory, J. Amer. Math. Soc. 9 (1996), no. 2, 473-527, DOI 10.1090/S0894-0347-96-00192-0. MR1322847

[BK09a] Jonathan Brundan and Alexander Kleshchev, Blocks of cyclotomic Hecke algebras and Khovanov-Lauda algebras, Invent. Math. 178 (2009), no. 3, 451-484, DOI 10.1007/s00222-009-0204-8. MR2551762

[BK09b] Jonathan Brundan and Alexander Kleshchev, Graded decomposition numbers for cyclotomic Hecke algebras, Adv. Math. 222 (2009), no. 6, 1883-1942, DOI 10.1016/j.aim.2009.06.018. MR 2562768

[BKW11] Jonathan Brundan, Alexander Kleshchev, and Weiqiang Wang, Graded Specht modules, J. Reine Angew. Math. 655 (2011), 61-87, DOI 10.1515/CRELLE.2011.033. MR2806105

[CDW12] Matthias Christandl, Brent Doran, and Michael Walter, Computing multiplicities of Lie group representations, 2012 IEEE 53rd Annual Symposium on Foundations of Computer Science-FOCS 2012, IEEE Computer Soc., Los Alamitos, CA, 2012, pp. 639-648. MR 3186652

[CSW18] Matthias Christandl, M. Burak Şahinoğlu, and Michael Walter, Recoupling coefficients and quantum entropies, Ann. Henri Poincaré 19 (2018), no. 2, 385-410, DOI 10.1007/s00023-017-0639-1. MR.3748296

[CHM07] Matthias Christandl, Aram W. Harrow, and Graeme Mitchison, Nonzero Kronecker coefficients and what they tell us about spectra, Comm. Math. Phys. 270 (2007), no. 3, 575-585, DOI 10.1007/s00220-006-0157-3. MR2276458

[CM06] Matthias Christandl and Graeme Mitchison, The spectra of quantum states and the Kronecker coefficients of the symmetric group, Comm. Math. Phys. 261 (2006), no. 3, 789-797, DOI 10.1007/s00220-005-1435-1. MR2197548

[CMT04] Joseph Chuang, Hyohe Miyachi, and Kai Meng Tan, A v-analogue of Peel's theorem, J. Algebra 280 (2004), no. 1, 219-231, DOI 10.1016/j.jalgebra.2004.04.017. MR.2081930

[DF12] Craig J. Dodge and Matthew Fayers, Some new decomposable Specht modules, J. Algebra 357 (2012), 235-262, DOI 10.1016/j.jalgebra.2012.01.035. MR2905253

[DG18] Stephen Donkin and Haralampos Geranios, Decompositions of some Specht modules I, J. Algebra 550 (2020), 1-22, DOI 10.1016/j.jalgebra.2019.12.017. MR4053571 
[EG02] Pavel Etingof and Victor Ginzburg, Symplectic reflection algebras, Calogero-Moser space, and deformed Harish-Chandra homomorphism, Invent. Math. 147 (2002), no. 2, 243-348, DOI 10.1007/s002220100171. MR 1881922

[Fay04] Matthew Fayers, Reducible Specht modules, J. Algebra 280 (2004), no. 2, 500-504, DOI 10.1016/j.jalgebra.2003.09.053. MR2089249

[Fay05] Matthew Fayers, Irreducible Specht modules for Hecke algebras of type A, Adv. Math. 193 (2005), no. 2, 438-452, DOI 10.1016/j.aim.2004.06.001. MR2137291

[Fay10] Matthew Fayers, On the irreducible Specht modules for Iwahori-Hecke algebras of type A with $q=-1$, J. Algebra 323 (2010), no. 6, 1839-1844.

[FL09] Matthew Fayers and Sinéad Lyle, Some reducible Specht modules for Iwahori-Hecke algebras of type $A$ with $q=-1$, J. Algebra 321 (2009), no. 3, 912-933, DOI 10.1016/j.jalgebra.2008.11.006. MR2488560

[FL13] Matthew Fayers and Sinéad Lyle, The reducible Specht modules for the Hecke algebra $\mathcal{H}_{\mathbb{C},-1}\left(\mathfrak{S}_{n}\right)$, J. Algebraic Combin. 37 (2013), no. 2, 201-241, DOI 10.1007/s10801012-0360-6. MR3011340

[FS16] Matthew Fayers and Liron Speyer, Generalised column removal for graded homomorphisms between Specht modules, J. Algebraic Combin. 44 (2016), no. 2, 393-432, DOI 10.1007/s10801-016-0674-x. MR3533560

[GMT18] Eugenio Giannelli, John Murray, and Joan Tent, Alperin-McKay natural correspondences in solvable and symmetric groups for the prime $p=2$, Ann. Mat. Pura Appl. (4) 197 (2018), no. 4, 999-1016, DOI 10.1007/s10231-017-0712-x. MR3829558

[GGOR03] Victor Ginzburg, Nicolas Guay, Eric Opdam, and Raphaël Rouquier, On the category $\mathcal{O}$ for rational Cherednik algebras, Invent. Math. 154 (2003), no. 3, 617-651, DOI 10.1007/s00222-003-0313-8. MR.2018786

[HSTZ13] Gerhard Heide, Jan Saxl, Pham Huu Tiep, and Alexandre E. Zalesski, Conjugacy action, induced representations and the Steinberg square for simple groups of Lie type, Proc. Lond. Math. Soc. (3) 106 (2013), no. 4, 908-930, DOI 10.1112/plms/pds062. MR3056296

[Ike15] Christian Ikenmeyer, The Saxl conjecture and the dominance order, Discrete Math. 338 (2015), no. 11, 1970-1975, DOI 10.1016/j.disc.2015.04.027. MR3357782

[Jam78] G. D. James, The representation theory of the symmetric groups, Lecture Notes in Mathematics, vol. 682, Springer, Berlin, 1978. MR.513828

[JK] Gordon James and Adalbert Kerber, The representation theory of the symmetric group, Encyclopedia of Mathematics and its Applications, vol. 16, Addison-Wesley Publishing Co., Reading, Mass., 1981. With a foreword by P. M. Cohn; With an introduction by Gilbert de B. Robinson. MR644144

[JLM06] Gordon James, Sinéad Lyle, and Andrew Mathas, Rouquier blocks, Math. Z. 252 (2006), no. 3, 511-531, DOI 10.1007/s00209-005-0863-0. MR2207757

[JM96] Gordon James and Andrew Mathas, Hecke algebras of type A with $q=-1$, J. Algebra 184 (1996), no. 1, 102-158, DOI 10.1006/jabr.1996.0251. MR1402572

[JM97] Gordon James and Andrew Mathas, $A$ q-analogue of the Jantzen-Schaper theorem, Proc. London Math. Soc. (3) 74 (1997), no. 2, 241-274, DOI 10.1112/S0024611597000099. MR1425323

[JM99] Gordon James and Andrew Mathas, The irreducible Specht modules in characteristic 2, Bull. London Math. Soc. 31 (1999), no. 4, 457-462, DOI 10.1112/S0024609399005822. MR.1687552

[KL09] Mikhail Khovanov and Aaron D. Lauda, A diagrammatic approach to categorification of quantum groups. I, Represent. Theory 13 (2009), 309-347, DOI 10.1090/S10884165-09-00346-X. MR2525917

[Kly04] A. Klyachko, Quantum marginal problem and representations of the symmetric group, arXiv:0409113, (2004).

[KN10] Alexander Kleshchev and David Nash, An interpretation of the Lascoux-LeclercThibon algorithm and graded representation theory, Comm. Algebra 38 (2010), no. 12, 4489-4500, DOI 10.1080/00927870903386536. MR2764833

[KOW12] Masao Kiyota, Tetsuro Okuyama, and Tomoyuki Wada, The heights of irreducible Brauer characters in 2-blocks of the symmetric groups, J. Algebra 368 (2012), 329344, DOI 10.1016/j.jalgebra.2012.07.001. MR2955236 
[LLT96] Alain Lascoux, Bernard Leclerc, and Jean-Yves Thibon, Hecke algebras at roots of unity and crystal bases of quantum affine algebras, Comm. Math. Phys. 181 (1996), no. 1, 205-263. MR 1410572

[Los16] Ivan Losev, Proof of Varagnolo-Vasserot conjecture on cyclotomic categories $\mathcal{O}$, Selecta Math. (N.S.) 22 (2016), no. 2, 631-668, DOI 10.1007/s00029-015-0209-7. MR3477332

[LS17] Sammy Luo and Mark Sellke, The Saxl conjecture for fourth powers via the semigroup property, J. Algebraic Combin. 45 (2017), no. 1, 33-80, DOI 10.1007/s10801-016-0700z. MR3591371

[Lyl07] Sinéad Lyle, Some q-analogues of the Carter-Payne theorem, J. Reine Angew. Math. 608 (2007), 93-121, DOI 10.1515/CRELLE.2007.054. MR.2339470

[Mur80] Gwendolen Murphy, On decomposability of some Specht modules for symmetric groups, J. Algebra 66 (1980), no. 1, 156-168, DOI 10.1016/0021-8693(80)90117-9. MR.591250

[O76] Jørn B. Olsson, McKay numbers and heights of characters, Math. Scand. 38 (1976), no. 1, 25-42, DOI 10.7146/math.scand.a-11614. MR409625

[O93] Jørn B. Olsson, Combinatorics and representations of finite groups, Vorlesungen aus dem Fachbereich Mathematik der Universität GH Essen [Lecture Notes in Mathematics at the University of Essen], vol. 20, Universität Essen, Fachbereich Mathematik, Essen, 1993. MR1264418

[PPV16] Igor Pak, Greta Panova, and Ernesto Vallejo, Kronecker products, characters, partitions, and the tensor square conjectures, Adv. Math. 288 (2016), 702-731, DOI 10.1016/j.aim.2015.11.002. MR.3436397

[PP17] Igor Pak and Greta Panova, Bounds on certain classes of Kronecker and q-binomial coefficients, J. Combin. Theory Ser. A 147 (2017), 1-17, DOI 10.1016/j.jcta.2016.10.004. MR3589885

[Rou08a] R. Rouquier, 2-Kac-Moody algebras, arXiv:0812.5023, 2008.

[Rou08b] Raphaël Rouquier, $q$-Schur algebras and complex reflection groups (English, with English and Russian summaries), Mosc. Math. J. 8 (2008), no. 1, 119-158, 184, DOI 10.17323/1609-4514-2008-8-1-119-158. MR2422270

[RSVV16] Raphaël Rouquier, Peng Shan, Michela Varagnolo, and Eric Vasserot, Categorifications and cyclotomic rational double affine Hecke algebras, Invent. Math. 204 (2016), no. 3, 671-786, DOI 10.1007/s00222-015-0623-7. MR3502064

[Spe14] Liron Speyer, Decomposable Specht modules for the Iwahori-Hecke algebra $\mathcal{H}_{\mathbb{F},-1}\left(\mathfrak{S}_{n}\right)$, J. Algebra 418 (2014), 227-264, DOI 10.1016/j.jalgebra.2014.07.011. MR 3250450

[Sta00] Richard P. Stanley, Positivity problems and conjectures in algebraic combinatorics, Mathematics: frontiers and perspectives, Amer. Math. Soc., Providence, RI, 2000, pp. 295-319. MR 1754784

[SW11] C. Stroppel and B. Webster, Quiver Schur algebras and q-Fock space, arXiv:1110.1115 2011.

[VV99] Michela Varagnolo and Eric Vasserot, On the decomposition matrices of the quantized Schur algebra, Duke Math. J. 100 (1999), no. 2, 267-297, DOI 10.1215/S0012-709499-10010-X. MR.1722955

[Web13] Ben Webster, Rouquier's conjecture and diagrammatic algebra, Forum Math. Sigma 5 (2017), Paper No. e27, 71, DOI 10.1017/fms.2017.17. MR3732238

Institut für Algebra, Zahlentheorie und Diskrete Mathematik, Leibniz Universität Hannover, D-30167 Hannover, Germany

Email address: bessen@math.uni-hannover.de

Department of Mathematics, University of York, Heslington, York, YO10 5DD, UNITED KINGDOM

Email address: Chris.Bowman-Scargill@york.ac.uk

Okinawa Institute of Science and Technology, Okinawa, Japan 904-0495

Email address: louise.sutton@oist.jp 